\title{
SCATTERING RESONANCES OF MICROSTRUCTURES AND HOMOGENIZATION THEORY*
}

\author{
S. E. GOLOWICH ${ }^{\dagger}$ AND M. I. WEINSTEIN ${ }^{\ddagger}$
}

\begin{abstract}
Scattering resonances are the eigenvalues and corresponding eigenmodes which solve the Schrödinger equation $H \psi=E \psi$ for a Hamiltonian, $H$, subject to the condition of outgoing radiation at infinity. We consider the scattering resonance problem for potentials which are rapidly varying in space and are not necessarily small in a pointwise sense. Such potentials are of interest in many applications in quantum, electromagnetic, and acoustic scattering, where the environment consists of microstructure, e.g., rapidly varying material properties. Of particular interest in applications are high contrast microstructures, e.g., large pointwise variations of material properties.

We develop a perturbation theory for the scattering resonances and eigenvalues of such high contrast and oscillatory potentials. The expansion is proved to be convergent in a norm which encodes the degree of oscillation in the microstructure. Next, we consider the concrete example of two-dimensional microstructure potentials. These correspond, for example, to a class of photonic waveguides with transverse microstructures. The leading order behavior is given by the scattering resonances of a suitable averaged potential, as predicted by classical homogenization theory. We show that the next term in the expansion agrees with that given by a higher order homogenization multiple-scale expansion, with an error term determined by the regularity of the potential. The higher order corrections, which take into account the detailed microstructure, have been shown by the authors to be important for efficient and accurate numerical computation of radiation rates.
\end{abstract}

Key words. scattering frequency, scattering resonance, quasi mode, radiation, photonic crystal, leaky mode, homogenization, tunneling, eigenvalue perturbation theory

AMS subject classifications. 35P25, 35B27, 78A40, 78A48

DOI. $10.1137 / 030600850$

\section{Introduction and overview.}

1.1. Scattering resonances and microstructures. Consider the timeindependent Schrödinger equation

$$
H \psi=E \psi
$$

where $H$ is the Hamiltonian

$$
H=-\Delta+V(x) \text {. }
$$

Scattering resonances of $(1.1)$ are solutions $(E, \psi)$, with $\psi \neq 0$, which satisfy an outgoing radiation condition at spatial infinity. The condition of outgoing radiation is a non-self-adjoint boundary condition, and therefore the scattering resonance energies, $E$, are typically complex numbers. A precise formulation of this radiation condition and the scattering resonance problem is given in section 4 .

In this paper, we are interested in the scattering resonances for (1.1) in the case where the potential, $V(x)$, has a background (slowly varying or "mean") part, $V_{0}(x)$,

${ }^{*}$ Received by the editors September 5, 2003; accepted for publication (in revised form) July 12, 2004; published electronically March 3, 2005.

http://www.siam.org/journals/mms/3-3/60085.html

${ }^{\dagger}$ Mathematical Sciences Research, Bell Laboratories, Lucent Technologies, Murray Hill, NJ. Current address: MIT Lincoln Laboratory, Lexington, MA 02420 (golowich@ll.mit.edu).

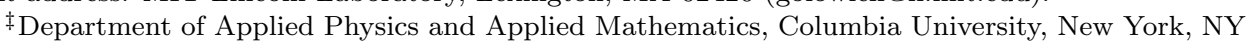
10027, and Mathematical Sciences Research, Bell Laboratories, Lucent Technologies, Murray Hill, NJ (miw2103@columbia.edu). 
and a rapidly oscillatory perturbation, $\delta V(x)$, which is not necessarily pointwise small:

$$
V(x)=V_{0}(x)+\delta V(x) .
$$

We call such potentials potentials with microstructure and refer to $\delta V(x)$ as the microstructure part of the potential. If the variations in the values of the potential are large, we refer to a high contrast potential. The Hamiltonian with potential $V_{0}(x)$ will be denoted $H_{0}$ and that with perturbed potential denoted $H$. A consequence of the results of this paper is a rigorous analytical foundation for the work in [7], where we derived a multiple-scale expansion for certain microstructured potentials, which arise as photonic microstructures, and used it as part of an accurate and efficient scheme for numerically computing scattering resonances; see section 1.2. A detailed discussion of the application to photonic microstructures, as well as an announcement of some of the current analytical work, is contained in [8].

The scattering resonance problem is of great mathematical interest and physical importance, arising in quantum, acoustic, elastic, and electromagnetic scattering. A basic problem in each field is to consider the situation of a compact region of space which is occupied by a collection of inhomogeneities or "scatterers," while outside this region the medium is homogeneous. Thus, outside this compact region the elementary propagating states are plane waves, $e^{i(k \cdot x-\omega t)}$, where $\omega=\omega(k)$ is the dispersion relation of the homogeneous medium $(k=|k|, k=2 \pi / \lambda, \lambda=$ wavelength). For a fixed wavevector, $k$, one considers a plane wave incident on a collection of inhomogeneities or "scatterers" and calculates the scattered field. The mapping from incident to scattered field is determined by the scattering matrix, $S(k)$. In many situations, $S(k)$ can be analytically continued from the positive real axis into the complex plane. Its poles in the lower half-plane are called resonances. Corresponding to these resonances are (non- $L^{2}$ ) states. Scattering resonances play an important role in the dynamics of waves. Namely, a general spatially localized initial condition incident on our collection of scatterers will typically interact for some time and give rise to a spray of radiation. The local energy, in a neighborhood of the scatterers, will die away as time evolves. The long-time transient exponential rate of local energy decay is determined by the imaginary parts of the resonances. In particular, one expects this decay to be limited by the resonance with the smallest imaginary part. For a given resonance, one refers to its imaginary part as the line-width or reciprocal lifetime of the associated state.

Being based on the Schrödinger equation (1.1), the analysis of this paper applies to a nonrelativistic quantum particle in a rapidly varying landscape [13], to the acoustic propagation of small amplitude pressure fluctuations about an equilibrium in an inhomogeneous medium [17], and to electromagnetic waves, in the scalar approximation, propagating in an inhomogeneous dielectric medium [26, 14].

Our initial motivation comes from the electromagnetic context, where there is a great deal of interest in the analysis of optical properties of media comprised of microor nanostructures. Recent advances in fabrication technology have made possible, through variations in material contrast and distribution of microfeatures, the manipulation of optical properties of composite media in a manner analogous to the way the electrical properties of materials have been manipulated for many years. Examples range from engineering the dispersion properties of photonic waveguides $[20,11]$ to cavity QED experiments, with a view toward applications to quantum computers [28]. Scattering resonances are of central importance in the behavior of such structures. The real parts are relatively insensitive to the detailed microstructure and are approximated by an averaged potential model, leading order homogenization 
theory. As illustrated below and extensively in [7], the imaginary parts are highly sensitive to the detailed microstructure, and we require higher order homogenization theory to estimate them accurately. Imaginary parts of scattering resonances of these structures correspond to the "Q-factor" of the effective cavity resonator.

1.2. Application to photonic waveguides. A specific example, considered in detail in [7], is an optical fiber waveguide with transverse microstructure. Such waveguides are often called photonic crystal fibers or holey fibers. In the scalar approximation, the transverse components of the electric field $\mathbf{E}_{\perp}=e^{i \beta x_{3}} \mathbf{e}_{\perp}\left(x_{1}, x_{2}\right)$ each satisfy the Helmholtz wave equation

$$
\left(\Delta+k^{2} n^{2}(x)-\beta^{2}\right) \psi=0 .
$$

Here, $\Delta$ denotes the Laplace operator with respect to the transverse variables $\left(x_{1}, x_{2}\right)$, $k=2 \pi / \lambda, \lambda$ denotes the wavelength of light, $n(x)$ denotes the refractive index profile, and $\beta$ denotes the propagation constant. Let $n_{g}$ denote a background refractive index. Then, the Helmholtz equation can be rewritten as a Schrödinger equation (1.1) with the following definitions:

$$
V(x)=k^{2}\left(n_{g}^{2}-n^{2}(x)\right), \quad E=k^{2} n_{g}^{2}-\beta^{2} .
$$

Here, $x$ denotes the two-dimensional spatial coordinate in the plane transverse to the waveguide.

A class of examples corresponding to a large family of microstructured waveguides of interest, treated in detail in section 6 , is

$$
V(x)=V_{N}(x)=V_{0}(|x|)+\delta V_{N}(x)=V_{0}(r)+\delta V(r, N \theta) .
$$

Here, $(r, \theta)$ are polar coordinates for $x \in \mathbb{R}^{2}$. The potential defined by (1.5) corresponds to a $N$-fold symmetric structure. The functions $V_{0}(r)$ and $\delta V(r, \Theta)$ are compactly supported locally $L^{2}$ functions. The parameter $N$ is a positive integer. When $N$ is large, the potential $V_{N}$ is a rapidly varying perturbation of $V_{0}$. This perturbation does not tend to zero pointwise but does tend to zero in a weak sense. Examples of such high contrast microstructure potentials appear in Figures 1 and 2.

We have derived and numerically implemented homogenization and corrected homogenization theories of scattering resonances and compared the results with direct numerical simulation [7]. Numerical results indicate that the real parts of scattering resonances are well approximated by those associated with the averaged potential, $V_{0}(r)$. However, the situation with imaginary parts of scattering resonances is very different. Figure 3 contrasts predictions for the imaginary parts of scattering resonances for structures of the type illustrated in Figure 1. Results for five different structures parametrized by $N$ and "fill fraction" $f$ are shown. The imaginary part of a scattering resonance is plotted as a function of a wavelength, $\lambda$. The crosses correspond to direct numerical simulation. The two curves correspond to predictions obtained from the average potential (dashed curve) and the second order corrected homogenization theory (solid curve), the validity of which is proved in this work; see the discussion below. Corrected homogenization gives excellent agreement with direct numerical simulations over wavelength ranges of interest in the particular application. For structures of the type shown in Figure 2, the corrected homogenization theory gives an even more dramatic improvement over the averaged (homogenization) theory. Clearly, and in general, numerical methods informed by analytical estimates on resonances can play an important role in finding optimal structures, e.g., maximizing "Q-factors." 


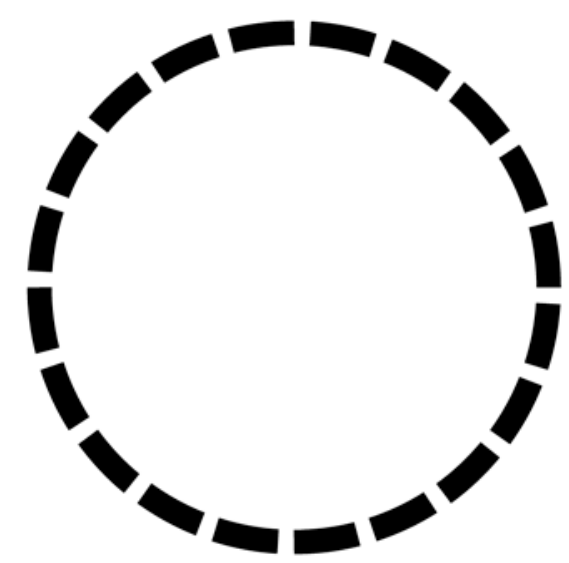

(a)

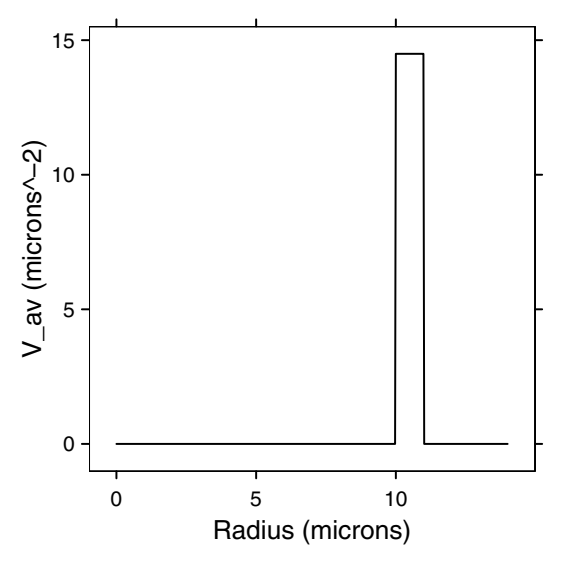

(b)

FIG. 1. (a) Black regions form the support of an $N$-fold symmetric potential $(N=20) . V(x)$ takes on one constant value on these regions and is zero outside. (b) The averaged potential $V_{0}(r)=$ $(2 \pi)^{-1} \int_{0}^{2 \pi} V(r, \Theta) d \Theta$, where $V(x)=V_{0}(r)+\delta V(r, N \theta), r=|x|$.

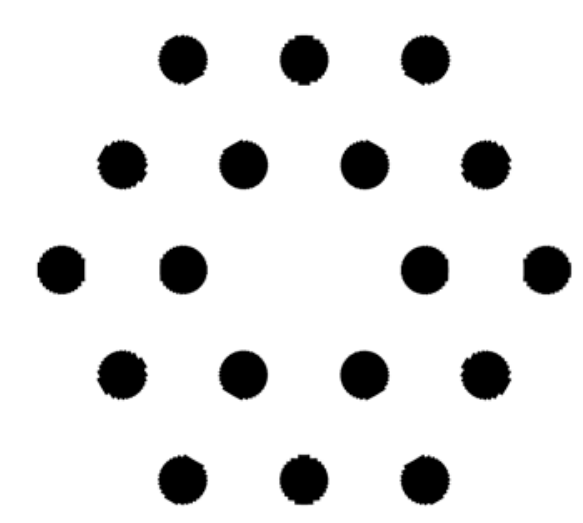

(a)

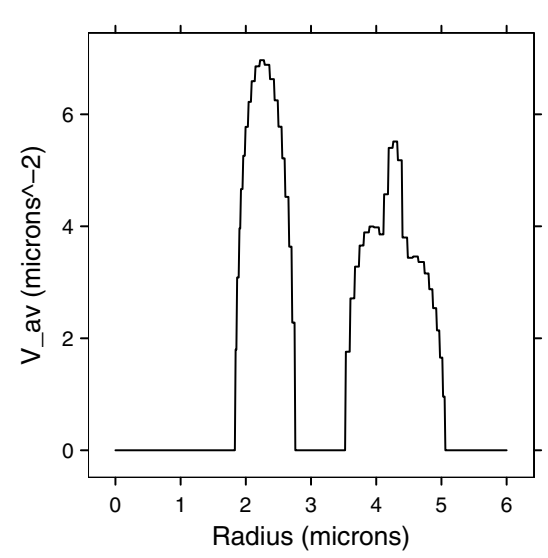

(b) $V_{0}(|x|)$.

FIG. 2. (a) An 18-hole subset of a hexagonal lattice $(N=6)$ with (b) the averaged potential

The current work implies analytical justification for the homogenization expansion and numerical approach summarized above and presented in detail in [7]. In particular, Theorems 4.1 and 7.1 imply the validity of second order homogenization, provided that a particular norm of the perturbation, $\left\|\delta V_{N}\right\|$, is sufficiently small. By Theorem 6.1, this smallness condition can be expressed as

$$
\frac{1}{\lambda^{2}} \cdot \frac{1}{N} \cdot C_{*} \text { sufficiently small, }
$$

where the constant $C_{*}=\sup _{M} M\left\|\delta V_{M}\right\| \|$ (see Theorem 6.1), which depends on the details of the microstructure. Our analysis applies to the case of fixed $\lambda$ and $N$ sufficiently large. However, the condition (1.6) suggests validity for fixed $N$ (fixed microstructure) and $\lambda$ large. Results which encompass this limit as well will be presented 


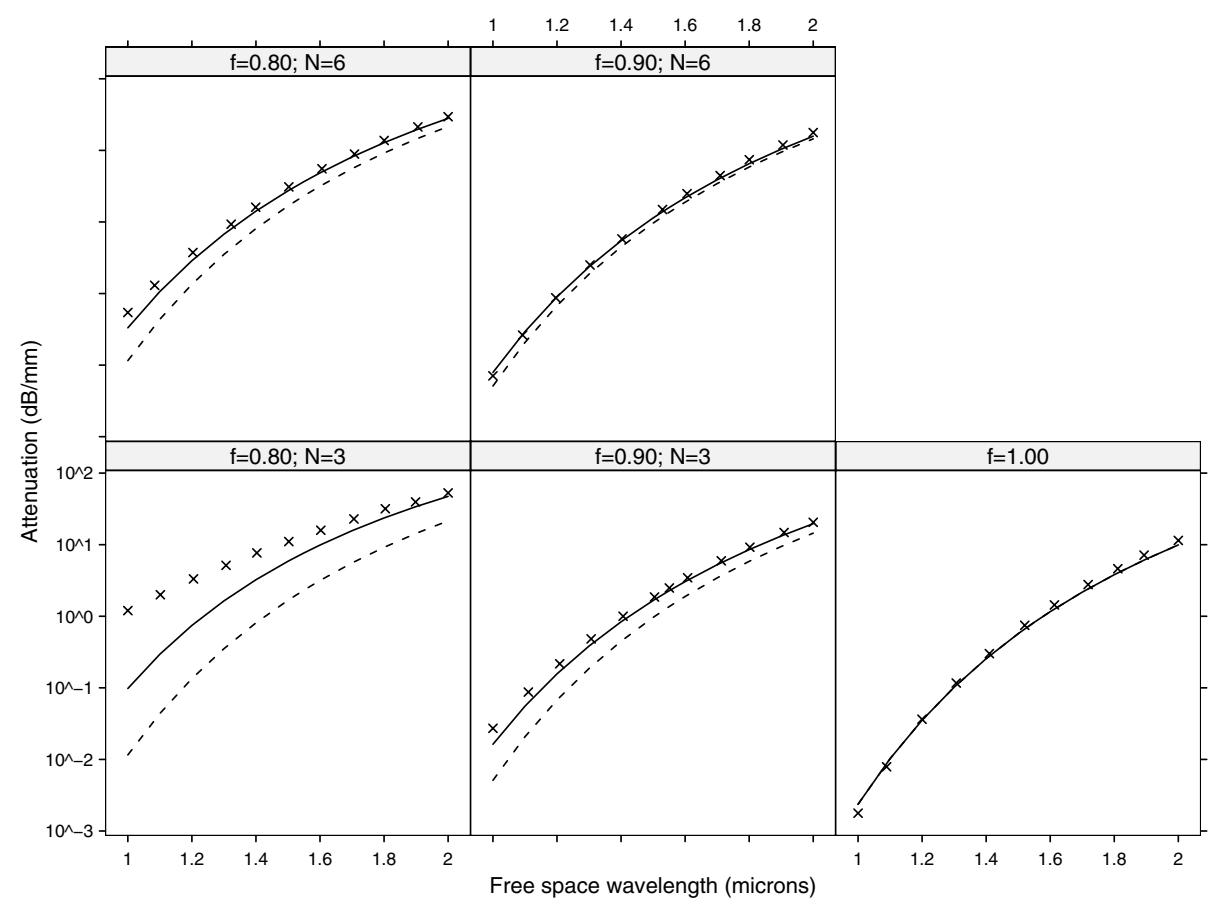

FIG. 3. Imaginary parts of scattering resonances, corresponding to scattering attenuation rate in the optical waveguide context, for the lowest order (fundamental or $L P_{01}$ ) resonance. Calculation is for $N$-fold symmetric structures of the type shown in Figure 1 for different fill fractions, $f$, and $N=3$ and $N=6$. The calculations were performed for a range of wavelengths, $\lambda$. Solid curves are attenuations computed using second order, $\mathcal{O}\left(N^{-2}\right)$, homogenization, proved herein to be valid for large $N$. Dashed curves correspond to leading order homogenization (averaging) theory. Crosses correspond to the results of direct numerical simulation presented in Figure 3(a) of [19].

in a separate publication.

We conclude this subsection with a discussion of two observations on departures of approximate theories from direct numerical simulation. (1) Note that as the wavelength is decreased, there is an increasing departure of approximate theoretical results from simulation results. The effect is most pronounced for the structure whose parameters are $N=3$ and $f=.8$. This trend can be understood by noting that as the wavelength is decreased with the structure held fixed, one enters the regime of geometrical optics, where the classical ray picture applies, rather than the wave picture, in which averaging can be expected to apply. Nevertheless, we find good agreement in our simulations for a ratio of wavelength to microfeature size of about $3 / 2$. (2) The plots in Figure 3 show a systematic underestimation of the "exact" imaginary parts. Note that there are two essential mechanisms of scattering loss: free space diffraction or ballistic propagation - propagation concentrated on rays where the potential is zero in between the barriers, where $V$ is positive; and tunneling - propagation along rays which impinge on regions where $V$ is positive. Homogenization theory replaces a problem in which both mechanisms are present by an effective problem, defined by the effective potential barrier, $V_{0}(|x|)$, for which tunneling is the only mechanism. Although, still rooted in homogenization ideas, our higher order theory gives a very significant improvement in the approximation even for relatively small values of $N$ 
and wavelength to microfeature size ratios. Though we have found a natural small parameter, $\|\delta V\|$, for measuring the "size" of the microstructure perturbation, we believe a related mode-dependent intrinsic parameter exists, which should give yet deeper insight. This is currently under investigation.

1.3. An illustrative and elementary example. In this subsection we present a simple example which motivates our approach to spectral problems with high contrast microstructure. We present only a sketch of the ideas. All analytical details are contained within our full implemention for the resonance problem, which is far more involved.

Consider the Schrödinger eigenvalue problem with periodic boundary conditions for an eigenfunction $u \in L^{2}\left(S^{1}\right)$ and an eigenvalue, $E$ :

$$
\begin{gathered}
\left(-\partial_{\theta}^{2}+V(\theta)\right) u(\theta)=E u(\theta), \\
u(0)=u(1), \quad \partial_{\theta} u(0)=\partial_{\theta} u(1) .
\end{gathered}
$$

Here, $V(\theta) \in L_{\text {periodic }}^{\infty}$. We rewrite (1.7) as the equivalent equation of LippmanSchwinger type:

$$
\begin{gathered}
\left(I-(1+E)\left(I-\partial_{\theta}^{2}\right)^{-1}\right) U+\mathcal{Q}_{V} U=0, \\
U \equiv\left(I-\partial_{\theta}^{2}\right)^{\frac{1}{2}} u \\
\mathcal{Q}_{V} U \equiv\left(I-\partial_{\theta}^{2}\right)^{-\frac{1}{2}} V\left(I-\partial_{\theta}^{2}\right)^{-\frac{1}{2}} U .
\end{gathered}
$$

Using the implicit function theorem, one can prove the following theorem.

THEOREM 1.1. Let $\left(U_{0}, E_{0}\right)$ be a solution of the eigenvalue problem (1.8) with potential $V_{0}$ and consider the perturbed eigenvalue problem with potential $V$. Let $\delta V \equiv V-V_{0}$ denote the perturbation. If the mapping $U \mapsto \mathcal{Q}_{\delta V} U$ has small norm as an operator from $L^{2}$ to $L^{2}$, i.e.,

$$
\left\|\left(I-\partial_{\theta}^{2}\right)^{-\frac{1}{2}} \delta V\left(I-\partial_{\theta}^{2}\right)^{-\frac{1}{2}}\right\|_{\mathcal{B}\left(L^{2}\right)} \text { sufficiently small, }
$$

then $\left(U_{0}, E_{0}\right)$ perturbs analytically to a nearby solution, $(U(V), E(V))$, of the eigenvalue problem.

Remark 1.2. While the operator norm in (1.9) can be small if $\delta V$ is pointwise small, e.g., $\delta V(\theta)=\varepsilon Q(\theta), 0<\varepsilon \ll 1$, it can also be small if $\delta V$ is pointwise large (high contrast) but sufficiently oscillatory (microstructure). For example, if $\delta V_{N}(\theta)=Q(N \theta)$, then the operator norm (1.9) is $\mathcal{O}\left(N^{-1}\right)$ by the following lemma (see also Theorem 6.1).

Lemma 1.3. Let $f(\theta)$ be a $2 \pi$ period function. Then,

$$
\begin{aligned}
& \text { the mapping } f(\theta) \mapsto e^{i N \theta} f(\theta) \text { has } L^{2} \text { norm one, } \\
& \text { the mapping } f(\theta) \mapsto\left\langle\partial_{\theta}\right\rangle^{-1} e^{i N \theta}\left\langle\partial_{\theta}\right\rangle^{-1} f(\theta) \text { has } L^{2} \text { norm } \mathcal{O}\left(N^{-1}\right) .
\end{aligned}
$$

\subsection{Goals, results, and overview.}

Perturbation problem. Suppose the $\left(E_{0}, \psi_{0}\right)$ is a solution to the scattering resonance problem for the Hamiltonian $H_{0}$ with potential $V_{0}(x)$. Our goals are to address the following issues.

(1) For $\delta V(x)$ sufficiently oscillatory (has sufficiently fine microstructure) and high contrast, show that the scattering resonance problem for $H$ with potential $V(x)=$ $V_{0}(x)+\delta V(x)$ has a "nearby" solution, $(E(V), \psi(V))$. 
(2) Develop a resonance perturbation theory in terms of a natural parameter, measuring the fineness of the microstructure.

(3) Relate (1) and (2) to homogenization theory.

In sections 3 and 4 we reformulate the scattering resonance problem as a "preconditioned" Lippman-Schwinger type equation (Theorem 3.2):

$$
\left(I+T_{R}(E) T_{V}\right) \Psi=0,
$$

where the operators $T_{R}(E)$ and $T_{V}$ are defined in section 3. We then obtain a stability and perturbation theory of scattering resonances in a norm appropriate for the study of microstructure perturbations which are not necessarily pointwise small (Theorem 4.1). The norm of the perturbation, $\|\delta V\| \|$, gives a natural measure of the degree of fineness of the microstructure and is defined for a compactly supported $L^{2}$ perturbation, $\delta V$, by

$$
\left\|T_{\delta V}\right\|_{\mathcal{B}\left(L^{2}\right)} \equiv\|\delta V\|=\left.\left\|\langle D\rangle^{-1} \delta \tilde{V}\langle D\rangle^{-1}\right\|\right|_{\mathcal{B}\left(L^{2}\right)}
$$

see also (4.1). Here, $\delta \tilde{V}=\chi^{-1} \delta V \chi^{-1}, \chi(x)=\mathcal{O}\left(e^{-\alpha|x|}\right)$, for some $\alpha>0$ and $\langle D\rangle=\left(m^{2}-\Delta\right)^{\frac{1}{2}}$.

If $\delta V$ is pointwise large but very oscillatory $\|\delta V V\|$ may be small due to the operator $\langle D\rangle^{-1}$, which gives small weight to the part of $\delta V$ supported at high wavenumbers; see the discussion of section 1.3. For the class of examples represented by (1.5) we have (Theorem 6.1)

$$
\left\|\delta V_{N}\right\|=\mathcal{O}\left(\frac{1}{N}\right)
$$

The main results of this paper are the following:

1. Theorem 3.2 introduces a characterization of solutions to the scattering resonance problem, (nonnormalizable) scattering resonance modes, and complex scattering resonance energies as solutions of a "preconditioned" Lippman-Schwinger equation defined on $L^{2}$.

2. Theorem 4.1 is a stability theorem showing that if the potential, $V_{0}$, is such that $\left(\psi\left(V_{0}\right), E\left(V_{0}\right)\right)$ is a (simple) scattering resonance pair and $V_{0}$ is perturbed to a "nearby" potential $V$, i.e., $\left\|V-V_{0}\right\| \mid$ is small, then the scattering resonance problem with potential $V$ has a nearby scattering resonance pair $(\psi(V), E(V))$. Furthermore, the mapping $V \mapsto(\psi(V), E(V))$ is analytic in $V$. The norm, \|\|$\cdot \|$, has been constructed so that if $V-V_{0}$ is not pointwise small but is very oscillatory, then $\left\|V-V_{0}\right\|$ is small. In section 4.2 we treat the case of degeneracies arising when $V_{0}$ is a radial potential, $V_{0}=V_{0}(r)$.

3. Theorem 7.1 demonstrates the connection with homogenization theory. For the class of microstructures, corresponding to high-contrast microstructures of the type arising in physical problems, we show that the $N^{-2}$ corrections to homogenization theory agree with the resonance perturbation theory of Theorem 4.1 with an error of order $N^{-2-\tau}, \tau>0$. The number $\tau$ depends on the regularity of the potential. In particular, if $V$ is $C^{2}$, then $\tau=2$, but if as is often the case in applications $V$ has jump discontinuities (e.g., an interface between two different materials at which there is a jump in refractive index), then $\tau=1$.

4. Theorems 2.1 and 2.2 encompass the (simpler) perturbation theory of eigenvalues for microstructure potentials. 
Although the problem of stability of eigenvalues for self-adjoint operators (see sections 1.3 and 2) has many of the structural features of the resonance problem, the resonance problem requires a much more technical treatment for the following reasons:

- While eigenvalues of a self-adjoint operator, $H$, are poles of the resolvent (Green's function) $(H-E)^{-1}$, scattering resonance energies are poles, $E(\Im E \neq 0)$, of the analytic continuation of the resolvent across the continuous spectrum (branch cut) to a "nonphysical" sheet; see section 3 .

- Solutions of the eigenvalue equation $H \psi=E \psi$, where $E$ is a scattering resonance energy, do not lie in $L^{2}$ and in fact grow exponentially at infinity.

Overview.

- In section 2 we discuss microstructure perturbations of the eigenvalue problem.

- In section 3 we formulate the scattering resonance problem.

- In section 4 we state and prove our theorem on microstructure perturbations of scattering resonances.

- In section 5 we explicitly calculate the expansion of the scattering resonance pair $(E(V), \psi(V))$ about the case $V=V_{0}$.

- In section 6 we show that the theory applies to potentials of the form $V=$ $V_{0}(r)+\delta V(r, N \theta)$, where $V_{0}(r)$ supports scattering resonances and $N$ is sufficiently large.

- In section 7 the homogenization expansion of [7] is reviewed, and it is proved that the leading term and lowest nonzero correction in $N^{-1}$ agree with those of the expansion displayed in section 5 . As demonstrated in [7] the imaginary parts of scattering resonances (leakage rates) are very sensitive to the detailed microstructure. Therefore, it is important to understand the corrections to leading order (effective medium) homogenization theory. Our second order homogenization expansion leads, in examples of physical interest, to an efficient and accurate numerical method, giving very good agreement with full simulation by Fourier methods and multipole methods. The real parts of scattering resonances are far less sensitive to the specific microstructure, and often the first term in the expansion (the averaged or homogenized problem) gives a good approximation.

Finally, we wish to mention other work on spectral problems in the setting of microstructure. In [24] and [18] the validity of first order corrections to homogenized eigenvalues for divergence form (self-adjoint) elliptic operators with rapidly varying coefficients on bounded domains is analyzed. The problem of scattering resonances for the Helmholtz resonator problem is studied in $[5,6]$ and in other works cited therein.

1.5. Definitions, notation, and conventions. All integrals are assumed to be over all space $\left(\mathbb{R}^{n}\right)$ unless otherwise noted.

$(r, \theta)$ denote polar coordinates in $\mathbb{R}^{2}$

$\Re z$, the real part of $z$; $₹ z$, the imaginary part of $z$

Both $\bar{z}$ and $z^{*}$ are used to denote the complex conjugate of $z$.

$A^{*}$ denotes the adjoint of the operator $A$.

$[A, B]=A B-B A$, the commutator of $A$ and $B$.

The inner product for functions $f, g \in L^{2}\left(\mathbb{R}^{n}\right)$ is denoted by

$$
\langle f, g\rangle=\int \overline{f(x)} g(x) d x .
$$


For radial functions $f(r), g(r) \in L^{2}\left(\mathbb{R}^{n}\right)$ on $\mathbb{R}^{n}$ we denote the radial inner product by

$$
\langle f, g\rangle_{\mathrm{rad}}=\int_{0}^{\infty} \overline{f(r)} g(r) r^{n-1} d r .
$$

Fourier transform:

$$
\begin{aligned}
\mathcal{F}[g](\xi) & \equiv \hat{g}(\xi) \equiv \int e^{-i x \cdot \xi} g(x) d x, \\
g(x) & =\mathcal{F}^{-1}[\mathcal{F}[g]](x)=(2 \pi)^{-n} \int e^{i x \cdot \xi} \hat{g}(\xi) d \xi
\end{aligned}
$$

$\langle\xi\rangle=\left(m^{2}+\xi^{2}\right)^{1 / 2}, \xi^{2}=\xi \cdot \xi$.

$D=-i \nabla, \Delta=\nabla \cdot \nabla$, the Laplace operator.

Functional calculus:

$$
\begin{array}{r}
f(D) g \equiv \mathcal{F}^{-1}[f \mathcal{F}[g](\cdot)]=\frac{1}{(2 \pi)^{n}} \int e^{i x \cdot \xi} f(\xi) \hat{g}(\xi) d \xi, \\
\langle D\rangle g=\left(m^{2} I-\Delta\right)^{1 / 2} g=\frac{1}{(2 \pi)^{n}} \int e^{i x \cdot \xi}\langle\xi\rangle \hat{g}(\xi) d \xi .
\end{array}
$$

Exponentially localized weights: Let $0<\tilde{M}<M$.

$$
\begin{array}{ll}
\chi(x)=\mathcal{F}^{-1}[\hat{\chi}] \quad \text { and } & \tilde{\chi}(x)=\mathcal{F}^{-1}[\hat{\tilde{\chi}}], \quad \text { where } \\
\hat{\chi}(\xi)=\left(M^{2}+\xi^{2}\right)^{-4} & \text { and } \quad \tilde{\tilde{\chi}}(\xi)=\left(\tilde{M}^{2}+\xi^{2}\right)^{-4} .
\end{array}
$$

Note that $0<\chi(x)=\mathcal{O}\left(e^{-M|x|}\right)$ and $0<\tilde{\chi}(x)=\mathcal{O}\left(e^{-\tilde{M}|x|}\right)$. The exponential upper bound follows from deformation of the $\xi$ integral contour. Note that $\chi(x)$ is essentially the Bessel potential $G_{8}(x)$, which is strictly positive; see equation (26) in [27].

For a radial function, $g(r)$, defined on $\mathbb{R}^{2}$,

$$
f\left(D_{l}\right) g \equiv e^{-i l \theta} f(D) e^{i l \theta} g .
$$

$\left\langle D_{l}\right\rangle=\left(I-\Delta_{r}+\frac{l^{2}}{r^{2}}\right)^{\frac{1}{2}}$.

Young's inequality: Let $\alpha \star \beta$ denote the convolution of functions $\alpha$ and $\beta$.

$$
\|\alpha \star \beta\|_{L^{p}} \leq\|\alpha\|_{L^{1}}\|\beta\|_{L^{p}}, \quad p \geq 1 .
$$

Bessel functions: For any integer $\ell$,

$$
J_{\ell}(z)=\frac{i^{-\ell}}{\pi} \int_{0}^{\pi} e^{i z \cos \theta} \cos (\ell \theta) d \theta
$$

Fourier transform of uniform measure on $S^{n-1}$ : See, for example, [3]. For $n \geq 2$,

$$
\frac{1}{(2 \pi)^{\frac{n}{2}}} \int_{S^{n-1}} e^{i \omega \cdot z} d \omega=\frac{1}{|z|^{\frac{n-2}{2}}} J_{\frac{n-2}{2}}(|z|) .
$$


2. Microstructure perturbations of eigenvalues. We will study the timeindependent Schrödinger equation

$$
H \psi=E \psi,
$$

where

$$
H=-\Delta+V(x) .
$$

Throughout this paper, we require fairly stringent conditions on the potential, namely, that $V(x) \in L^{\infty}\left(\mathbb{R}^{n}\right)$ and $V(x)$ has compact support. Minor modifications in our arguments can be made to treat the case of potentials which decay exponentially as $|x| \rightarrow \infty$. For simplicity, we will work in spatial dimension $n=2$ or 3 , though we believe that generalization to higher dimensions can be done. Standard arguments $[22,9]$ imply that $H$ is self-adjoint on the domain $D(H)=D(-\Delta)=H^{2}\left(\mathbb{R}^{n}\right)$, and the essential spectrum $\sigma_{\text {ess }}(H)=\sigma(-\Delta)=[0, \infty)$.

In this section we focus on the eigenvalue problem for (2.1), requiring $\psi \in L^{2}$ and for the remainder of the paper the scattering resonance problem, corresponding to $(2.1)$, with $\psi$ "outgoing" at $\infty$.

The class of potentials we consider can be written as

$$
V(x)=V_{0}(x)+\delta V(x),
$$

where $V_{0}$ is "slowly varying" and $\delta V$ is a perturbation which is "rapidly varying," but possibly a pointwise large perturbation, in a sense which is made precise below.

A solution to the eigenvalue problem

$$
(-\Delta+V) \psi=E \psi
$$

is a pair $(E, \psi)$, where $\psi$ is nontrivial and $\psi \in H^{2}\left(\mathbb{R}^{n}\right)$.

We now reformulate the eigenvalue problem in a manner which is well suited to treating microstructure perturbations; see section 1.3. Let $E=\mu^{2}$. For $\Im \mu>0$ $(E \notin[0, \infty)),\left(-\Delta-\mu^{2}\right)^{-1}$ is bounded on $L^{2}$, and so (2.2) implies that

$$
\psi+\left(-\Delta-\mu^{2}\right)^{-1} V \psi=0
$$

or, equivalently,

$$
(I+\underbrace{\left(-\Delta-\mu^{2}\right)^{-1}(I-\Delta)}_{S_{R}(\mu)} \underbrace{\langle D\rangle^{-1} V\langle D\rangle^{-1}}_{S_{V}})\langle D\rangle \psi=0 .
$$

Therefore, we have the following simple correspondence between solutions of (2.2) and a Lipmann-Schwinger-type integral equation.

THEOREM 2.1. The pair $\left(E=\mu^{2}, \psi\right)$ is a solution to the eigenvalue problem if and only if $(\mu, \tilde{\psi})$, where $\tilde{\psi}=\langle D\rangle \psi \in L^{2}$, solves

$$
\left(I+S_{R}(\mu) S_{V}\right) \tilde{\psi}=0 .
$$

If $E_{0}$ is an eigenvalue, then $E_{0}=\mu_{0}^{2}<0$ for some $\mu_{0}$ on the imaginary axis in the upper half-plane. We have the following perturbation theorem for simple eigenvalues. 
Theorem 2.2. (a) Let $V_{0}$ denote a potential for which $\left\|V_{0}\right\|_{1}$ is finite, where

$$
\left\|\left|V_{0}\right|\right\| \equiv\left\|S_{V_{0}}\right\|_{\mathcal{B}\left(L^{2}\right)}=\left\|\langle D\rangle^{-1} V_{0}\langle D\rangle^{-1}\right\|_{\mathcal{B}\left(L^{2}\right)} .
$$

Let $\left(E_{0}, \psi_{0}\right)$ denote a solution of the eigenvalue problem corresponding to the potential, $V_{0}(x)$, and assume that $E_{0}$ is a simple eigenvalue, for which $\tilde{\psi}_{0}=\langle D\rangle \psi_{0}$ correspondingly spans the null space of $I+S_{R}\left(\mu_{0}\right) S_{V_{0}}$. Assume that

$$
\left\langle S_{V_{0}} \tilde{\psi}_{0}, S_{R}^{\prime}\left(\mu_{0}\right) S_{V_{0}} \tilde{\psi}_{0}\right\rangle=\left\|R_{0}\left(\mu_{0}\right) V_{0} \psi_{0}\right\|_{2}^{2} \neq 0 .
$$

Then, there exists $\varepsilon_{0}$ such that for any potential $V$ satisfying $\left\|V-V_{0}\right\| \mid<\varepsilon_{0}$, there corresponds a unique solution $(E(V), \psi(V))$ of the eigenvalue problem which lies near $\left(E_{0}, \psi_{0}\right)$.

(b) The mapping

$$
V \mapsto(E, \psi) \in \mathbb{R}^{1} \times H^{2},
$$

which associates to a potential $V$ a solution $(E(V), \psi(V))$ of the eigenvalue problem, is analytic in the norm $\||V|\|$ in a neighborhood $\left\|V-V_{0}\right\| \mid<\varepsilon_{0}$ of $V_{0}$.

The proof of this theorem is a very much simplified version of the proof of the analogous result (Theorem 4.1) for the case of scattering resonances. We implement the ideas in the latter context. However, we do make a few remarks presently.

Remark 2.3. The proofs are based on the implicit function theorem. Recall that an eigenvalue $E_{0}$ of $-\Delta+V_{0}$ is such that $E_{0}=\mu_{0}^{2}$, where $\mu$ is purely imaginary and in the upper half-plane. Also, as can easily be seen using the Fourier transform, the operators $\partial_{\mu}^{j} S_{R}(\mu), j \geq 0$, are bounded in $L^{2}$ in a neighborhood of $\mu_{0}$. Therefore, one expects the continuity of eigenvalues in the norm for potentials varying in a neighborhood of $V_{0}$ in the norm (2.5).

Remark 2.4. The results worked out in sections 5-7 apply as well for the case of the eigenvalue problem of this section, with much simpler proofs. These include

1. a convergent perturbation expansion of eigenvalues in the $V-V_{0}$ (compare section 5);

2. results for the special case of $N$-fold symmetric perturbations, $V-V_{0}=$ $\delta \tilde{V}(r, N \theta)$ (compare section 6);

3. a homogenization/multiple-scale expansion of eigenvalues as well as comparison and agreement with the eigenvalue perturbation theory through second order in $N^{-1}$ (compare section 7 ).

\section{Formulation of the scattering resonance problem.}

3.1. Elementary review of resonances. Our particular interest is in the resonances of $H$, which we will construct as perturbations of the resonances of $H_{0}=$ $-\Delta+V_{0}$. Therefore, we must first consider the latter resonances. Their existence for the restricted class of potentials we consider is well known [2]. We will briefly review the arguments, as they will serve as a starting point for the proofs of our main results.

Proposition 3.1. The resolvent $R_{V_{0}}(\mu)=\left(-\Delta+V_{0}-\mu^{2}\right)^{-1}$ can be analytically continued from the half-plane $\Im \mu>0$ to the entire plane $\mathbb{C}$, when $n$ is odd, or to the logarithmic Riemann surface $\Lambda$ when $n$ is even. The analytic continuation of the resolvent is meromorphic in $\mu$, with residues at the poles corresponding to finite-rank operators associated with nontrivial solutions of

$$
\left(-\Delta+V_{0}-\mu^{2}\right) u=0
$$




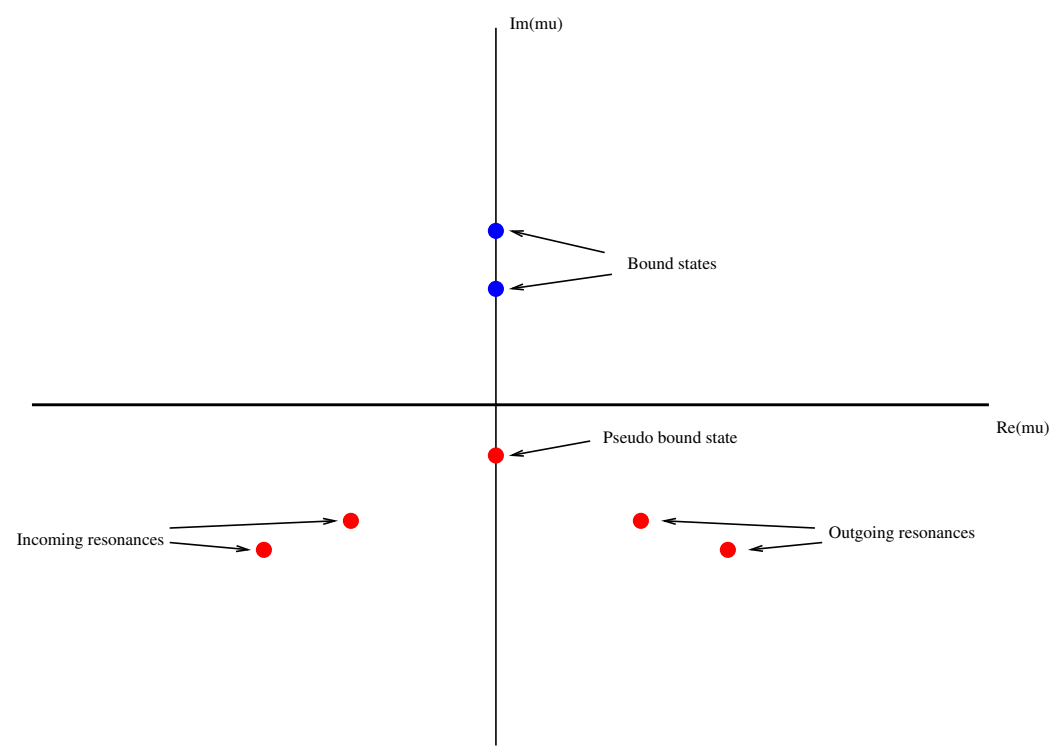

FIG. 4. Complex $\mu$ plane. Outgoing/incoming resonances correspond to solutions satisfying outgoing/incoming radiation conditions at infinity.

that satisfy either outgoing (+) or incoming (-) boundary conditions at infinity [25]:

$$
u(r \omega)=r^{-\frac{n-1}{2}} e^{ \pm i \mu r}[h(\omega)+o(1)] \text { as } r \rightarrow \infty,
$$

where $h$ is a continuous function on the unit sphere $S^{n-1}$.

The half-plane $\Im \mu>0$ is often called the "physical sheet" and $\Im \mu<0$ the "unphysical sheet." As seen below, poles of $R_{V_{0}}(\mu)$ on the physical sheet correspond to eigenvalues, $E=\mu^{2}<0(\Re \mu=0, \Im \mu>0)$. Those on the unphysical sheet correspond to outgoing $(\Re \mu>0, \Im \mu<0)$ or incoming $(\Re \mu<0, \Im \mu<0)$ solutions, which are not $L^{2}$. These are called resonances. See Figure 4.

Proof of Proposition 3.1. For $\Im \mu>0$, introduce the free resolvent $R_{0}(\mu)=$ $\left(-\Delta-\mu^{2}\right)^{-1}$. The kernel of the free resolvent $R_{0}(\mu)$ is known explicitly [16]:

$$
R_{0}(\mu ; x, y)=\frac{1}{4 i}\left(\frac{\mu}{2 \pi|x-y|}\right)^{\frac{n-2}{2}} H_{\frac{n-2}{2}}^{(1)}(\mu|x-y|),
$$

where $H_{\frac{n-2}{2}}^{(1)}$ is a Hankel function [1]. In odd dimensions $n \geq 3$, this Hankel function is entire as a function of $\mu$, while in even dimensions it is entire on the logarithmic covering $\Lambda$ of $\mathbb{C}$. The large $|z|$ asymptotics of Hankel functions are displayed in Appendix A. $H_{\nu}^{(1)}(z)$ satisfies an outgoing radiation condition as $|z| \rightarrow \infty$.

It is shown in [10] that in the physical upper half-plane, $\Im \mu>0$, the resolvent $R_{V_{0}}$ is meromorphic, with poles possibly existing on the imaginary axis. Poles, $\mu$, in the upper half-plane correspond to eigenvalues, $E=\mu^{2}<0$, of $H_{0}$. Away from its poles, the resolvent $R_{V_{0}}(\mu)$ satisfies

$$
\left(-\Delta+V_{0}-\mu^{2}\right) R_{V_{0}}(\mu)=I,
$$

which, since the domains of $-\Delta+V_{0}$ and $\Delta$ are equal, can be rewritten as

$$
\left(I+R_{0}(\mu) V_{0}\right) R_{V_{0}}(\mu)=R_{0}(\mu) .
$$


In order to analytically continue $R_{V_{0}}(\mu)$ from the physical half-plane to $\Im \mu<0$, it is evident from (3.3) and the asymptotic behavior of $H_{\nu}^{(1)},(\mathrm{A} .4)$, that we will need to work in a larger Hilbert space, since $H^{(1)}(\mu|x-y|)$ maps the space of square integrable functions that are compactly supported in $|x| \leq r_{0}, L_{c}^{2}\left(r_{0}\right)$, to $e^{\alpha|x|} L^{2}\left(\mathbb{R}^{n}\right)$, with $\alpha>|\Im \mu|$. It is possible to formulate the analytic continuation in $L^{2}\left(\mathbb{R}^{n}\right)$ by explicitly introducing localizing functions $\chi(x)$ satisfying

$$
0<\chi(x)=\mathcal{O}\left(e^{-\alpha|x|}\right) ;
$$

see (1.15). Multiplying (3.4) on the left by $\chi(x)$, we obtain

$$
\left(I+\chi R_{0}(\mu) V_{0} \chi^{-1}\right) \chi R_{V_{0}}(\mu)=\chi R_{0}(\mu) .
$$

Both sides of (3.6) are considered as mappings from $L_{c}^{2}\left(\mathbb{R}^{n}\right)$, the space of compactly supported $L^{2}$ functions, to $L^{2}\left(\mathbb{R}^{n}\right)$.

For $|\Im \mu|<\alpha$, it can be shown by direct estimation of the kernel associated with the operator $\chi R_{0} V_{0} \chi^{-1}$ using (3.3) that [2]

$$
\chi R_{0}(\mu) V_{0} \chi^{-1}: L^{2}\left(\mathbb{R}^{n}\right) \rightarrow L^{2}\left(\mathbb{R}^{n}\right)
$$

is Hilbert-Schmidt and therefore compact. Furthermore, it is also analytic in this region, and its norm tends to zero as $\Im \mu \rightarrow+\infty$ in the physical sheet. Therefore, the analytic Fredholm theorem [21] tells us that $\left(I+\chi R_{0} V_{0} \chi^{-1}\right)^{-1}$ is meromorphic in the domain of analyticity of $\mu$ and that the residues at the poles are finite-rank operators. From (3.6) and (3.3) is it clear that $\chi R_{V_{0}}(\mu)$ inherits the analyticity of $\left(I+\chi R_{0}(\mu) V_{0} \chi^{-1}\right)^{-1} \chi R_{0}(\mu)$.

Furthermore, if $\mu$ is at a pole, then

$$
\left(I+\chi R_{0} V_{0} \chi^{-1}\right) F_{0}=0
$$

has a nontrivial solution $F_{0} \in L^{2}\left(\mathbb{R}^{n}\right)$. Setting $\psi_{0}=\chi^{-1} F_{0}$, we see that

$$
\psi_{0}=-R_{0} V_{0} \psi_{0}
$$

which is equivalent to (3.1). Also, if the analytic continuation in $\mu$ is from the physical half-plane to the fourth quadrant across the positive real axis, then (3.8), along with the asymptotic behavior (A.4) of the Hankel function in (3.3), allows us to conclude that the solution is outgoing at infinity; if the continuation is across the negative real axis to the third quadrant, then it is incoming.

3.2. Resonances of microstructure potentials. We can now begin our study of resonances of microstructured potentials $V=V_{0}+\delta V$. The guiding intuition is that a resonance pair $\left(E_{0}, \psi_{0}\right)$ of $V_{0}$ should perturb to a nearby pair $(E, \psi)$ of $V$, if $\delta V$ is sufficiently oscillatory, depending on the pair $(E, \psi)$. To make these statements precise, we take (3.7) as the starting point. We assume that $\left(\mu^{2}, \Psi\right)$ are a resonance pair, with $\Psi \in L^{2}\left(\mathbb{R}^{2}\right)$, and write $\psi=\chi^{-1} \Psi$. We then introduce the smoothing operator $\langle D\rangle^{-1}$ and its inverse, $\langle D\rangle$, given in (1.14) to obtain

$$
(I+\underbrace{\langle D\rangle \chi R_{0}(\mu) \chi\langle D\rangle}_{T_{R}(\mu)} \underbrace{\langle D\rangle^{-1} \chi^{-1} V \chi^{-1}\langle D\rangle^{-1}}_{T_{V}})(\langle D\rangle \chi \psi)=0 .
$$


Theorem 3.2. Resonances are solutions $(\Psi, \mu)$, with $0 \neq \Psi \in L^{2}$, and $\Im \mu<0$ of

$$
\left(I+T_{R}(\mu) T_{V}\right) \Psi=0,
$$

where $T_{R}(\mu)$ and $T_{V}$ are defined in (3.9).

Remark 3.3. (1) The properties of $T_{R}(\mu)$ and $T_{V}$, which validate (3.10) as an alternative formulation of the resonance problem, are stated and proved below. In particular, we shall prove (Corollary 3.6) that $T_{V}(\mu) T_{V}$ is compact for $\Im \mu>-\tilde{M}$. By the analytic Fredholm theorem [23], $\left(I+T_{R}(\mu) T_{V}\right)^{-1}$ is meromorphic for $\Im \mu>-\tilde{M}$ with poles which are finite-rank operators. Poles in the lower half-plane are scattering resonances.

(2) The localizing operators $\chi$ are used to transfer decay from the potential to localization of the free resolvent, which facilitates analytic continuation in $\mu$ to the lower half-plane, where resonance energies are found.

(3) The operators $\langle D\rangle^{-1}$ and $\langle D\rangle$ transfer smoothness from the free resolvent $R_{0}$ to act on the microstructured potential $V$. This latter property enables the perturbative treatment of high contrast microstructures, as explained in section 1.3.

To prove Theorem 3.2 and to work with the formulation (3.10) we require the following two lemmas.

Lemma 3.4. The operator

$$
T_{V}: L^{2}\left(\mathbb{R}^{n}\right) \rightarrow L^{2}\left(\mathbb{R}^{n}\right)
$$

is Hilbert-Schmidt, and therefore compact, for $n \leq 3$.

Lemma 3.5. Assume $n \leq 3$, and let $k \geq 0$ be arbitrary. The operator

$$
\partial_{\mu}^{k} T_{R}(\mu): L^{2}\left(\mathbb{R}^{n}\right) \rightarrow L^{2}\left(\mathbb{R}^{n}\right)
$$

is defined, bounded and analytic in $\mu$ for $\Im \mu>-\tilde{M}>-m, m>0$.

Corollary 3.6. The operator

$$
T_{R}(\mu) T_{V}: L^{2}\left(\mathbb{R}^{n}\right) \rightarrow L^{2}\left(\mathbb{R}^{n}\right)
$$

is compact and analytic in $\mu$ for $\Im \mu>-\tilde{M}>-m$ and $n \leq 3$.

Proof of Lemma 3.4. Let

$$
\tilde{V}=\chi^{-1} V \chi^{-1}
$$

Note that $\tilde{V}$ has the same support as $V$. To prove that $T_{V}$ is compact, note that

$$
\langle D\rangle^{-1} \tilde{V}\langle D\rangle^{-1} f=\int K(x, y) f(y) d y,
$$

where

$$
K(x, y)=\int \frac{d \xi}{(2 \pi)^{n}} \frac{d \eta}{(2 \pi)^{n}} e^{i(x \cdot \xi-y \cdot \eta)} \frac{\hat{\tilde{V}}(\xi-\eta)}{\langle\xi\rangle\langle\eta\rangle}
$$

where $\langle D\rangle^{-1}=\left(m^{2}-\Delta\right)^{-1 / 2}$ and $\langle\xi\rangle=\left(m^{2}+\xi^{2}\right)^{1 / 2}$. We claim that

$$
\iint|K(x, y)|^{2} d x d y<\infty
$$


implying that $T_{V}$ is a Hilbert-Schmidt operator and therefore compact on $L^{2}$.

We now verify (3.13). The Fourier transform of $K(x, y)$ is given by

$$
\hat{K}(\xi, \eta)=\frac{\hat{\tilde{V}}(\xi+\eta)}{\left(m^{2}+|\xi|^{2}\right)^{\frac{1}{2}}\left(m^{2}+|\eta|^{2}\right)^{\frac{1}{2}}} .
$$

By the Plancherel theorem

$$
\begin{aligned}
\int d x d y|K(x, y)|^{2} & =(2 \pi)^{2 n} \int d \xi d \eta|\hat{K}(\xi, \eta)|^{2} \\
& =(2 \pi)^{2 n} \int d \xi d \eta \frac{|\hat{\tilde{V}}(\xi+\eta)|^{2}}{\left(m^{2}+|\xi|^{2}\right)\left(m^{2}+|\eta|^{2}\right)} \\
& =(2 \pi)^{2 n} \int \frac{1}{\left(m^{2}+|\xi|^{2}\right)} d \xi \int|\hat{\tilde{V}}(\eta+\xi)|^{2} \frac{1}{\left(m^{2}+|\eta|^{2}\right)} d \eta
\end{aligned}
$$

The inner integral $(d \eta)$ is the convolution of an $L^{1}$ function, $|\hat{\tilde{V}}|^{2}$, and, for dimension $n<4$, an $L^{2}$ function, $\langle\eta\rangle^{-2}$, and is therefore in $L^{2}(d \eta)$ by Young's inequality, (1.16). It follows that the integrand of the outer integral $(d \xi)$ is the product of $L^{2}$ functions and by the Cauchy-Schwarz inequality

$$
\int d x d y|K(x, y)|^{2} \leq(2 \pi)^{2 n}\left\|\langle\xi\rangle^{-2}\right\|_{2}^{2}\|\tilde{V}\|_{2}^{2}<\infty .
$$

Lemma 3.4 now follows.

The following lemma, concerning analyticity properties of the free resolvent kernel, is central to analytic extension of $T_{R}(\mu)$ into the lower half-plane and the proof of Lemma 3.5.

Lemma 3.7. Let $n \geq 2$. For $j=0,1$, the operator

$$
\mathcal{L}_{j}(\mu)=R_{0}(\mu)\langle D\rangle^{j}
$$

has a kernel $\mathcal{L}_{j}(\mu ; x, y)$ that can be analytically continued from the (physical) upper half-plane to the lower half-plane, with a branch cut starting at-im. The kernel is given by

$\mathcal{L}_{j}(\mu ; x, y)=m^{j+n-2} G_{2-j}(m(x-y))+\left(m^{2}+\mu^{2}\right) m^{j+n-4} G_{4-j}(m(x-y))+\mathcal{R}_{j}(\mu ; x, y)$.

Here, $G_{\alpha}(x)$ denotes the kernel associated with the Bessel potential $(I-\Delta)^{-\frac{\alpha}{2}}$, defined in terms of the Fourier transform [27]

$$
\hat{G}_{\alpha}(\xi)=\left(m^{2}+\xi^{2}\right)^{-\frac{\alpha}{2}} .
$$

For $\mu$ in the physical upper half-plane, $\mathcal{R}_{j}$ is defined by

$$
\mathcal{R}_{j}(\mu ; x, y)=\frac{\left(m^{2}+\mu^{2}\right)^{2}}{(2 \pi)^{n}} \int e^{i \xi \cdot(x-y)} \frac{d \xi}{\left(\xi^{2}-\mu^{2}\right)\left(m^{2}+\xi^{2}\right)^{2-j / 2}} .
$$

The first two terms in (3.18) form a quadratic polynomial in $\mu$ and is therefore an entire function of $\mu$. The analytic continuation of $\mathcal{R}_{j}(\mu ; x, y)$ from $\Im \mu>0$ to $\Im \mu<0$ 
is given by

$$
\mathcal{R}_{j}(\mu ; x, y)=\mathcal{R}_{j}(-\mu ; x, y)+\left(m^{2}+\mu^{2}\right)^{\frac{j}{2}} \frac{\pi i}{(2 \pi)^{n}}\left(\frac{\mu}{|x-y|}\right)^{\frac{n-2}{2}} J_{\frac{n-2}{2}}(\mu|x-y|),
$$

where $J_{\alpha}$ is the Bessel function [1]. Furthermore, $\mathcal{R}_{j}(\mu ; x, y)$ satisfies

$$
\mathcal{R}_{j}(\mu ; x, y)=\left(m^{2}+\mu^{2}\right)^{\frac{j}{2}} R_{0}(\mu ; x, y)+\mathcal{R}_{j}^{(2)}(\mu ; x-y),
$$

where $R_{0}(\mu ; x, y)$ denotes the free resolvent kernel given by (3.3) and

$$
\left|\partial_{\mu}^{k} \mathcal{R}_{j}^{(2)}(\mu ; x, y)\right| \leq C(b, k) e^{-b|x-y|}, \quad \Im \mu \geq 0,
$$

for any $b<m$ and any $k \geq 0$.

Proof of Lemma 3.7. For $\Im \mu>0$ we represent the kernel of $\mathcal{L}_{j}(\mu)$ using the Fourier transform:

$$
\mathcal{L}_{j}(\mu ; x, y)=\int \frac{d \xi}{(2 \pi)^{n}} e^{i \xi \cdot(x-y)} \frac{\left(m^{2}+\xi^{2}\right)^{j / 2}}{\xi^{2}-\mu^{2}} .
$$

Using the algebraic identity

$$
\frac{1}{\xi^{2}-\mu^{2}}=\frac{1}{m^{2}+\xi^{2}}+\frac{m^{2}+\mu^{2}}{\left(\xi^{2}-\mu^{2}\right)\left(m^{2}+\xi^{2}\right)},
$$

we have

$$
\begin{aligned}
\mathcal{L}_{j}(\mu ; x, y)=\int \frac{d \xi}{(2 \pi)^{n}} e^{i \xi \cdot(x-y)}[ & \frac{1}{\left(m^{2}+\xi^{2}\right)^{1-j / 2}}+\frac{\left(m^{2}+\mu^{2}\right)}{\left(m^{2}+\xi^{2}\right)^{2-j / 2}} \\
& \left.+\frac{\left(m^{2}+\mu^{2}\right)^{2}}{\left(\xi^{2}-\mu^{2}\right)\left(m^{2}+\xi^{2}\right)^{2-j / 2}}\right] .
\end{aligned}
$$

The expansion (3.18) now follows from definition (3.19).

The first two terms of (3.18) are entire in $\mu$. To analytically continue the remaining term $\mathcal{R}_{j}(\mu ; x, y)$ we will adapt an argument in [15]. We begin by rewriting $\mathcal{R}_{j}$ as

$$
\mathcal{R}_{j}(\mu ; x, y)=\frac{\left(m^{2}+\mu^{2}\right)^{2}}{(2 \pi)^{n}} \int_{S^{n-1}} d \omega \int_{0}^{\infty} d \rho \rho^{n-1} e^{i \rho \omega \cdot(x-y)} \frac{1}{\left(\rho^{2}-\mu^{2}\right)\left(m^{2}+\rho^{2}\right)^{2-j / 2}} .
$$

We now focus on the $\rho$ integral. Its integrand is analytic in $\rho$ except for poles at $\rho= \pm \mu$ and, for the case of $j=1$, a branch cut starting at $\rho= \pm i m$. Let $\mu_{1}=\mu^{\prime}+i \epsilon_{1}$ and $\mu_{2}=-\mu^{\prime}+i \epsilon_{2}$, where $\mu^{\prime} \in \mathbb{R}_{+}$and $\epsilon_{j}>0$, denote points in the first and second quadrants. We begin by considering $\mathcal{R}\left(\mu_{1} ; x, y\right)$ and $\mathcal{R}\left(\mu_{2} ; x, y\right)$ separately. $\mathcal{R}\left(\mu_{1} ; x, y\right)$ can be expressed as an integral over the contour in Figure 5, part of which is the lower half of a circle about $\mu^{\prime}$ traversed counterclockwise, $\gamma_{-}\left(\mu^{\prime}\right)$. $\mathcal{R}\left(\mu_{2} ; x, y\right)$ can be expressed as an integral over the contour in Figure 6 , part of which is the upper half of a circle about $\mu^{\prime}$ traversed clockwise, $\gamma_{+}\left(\mu^{\prime}\right)$. In this representation of $\mathcal{R}\left(\mu_{2} ; x, y\right)$, we let $\epsilon_{2}$ approach $-\epsilon_{1}\left(\mu_{2} \rightarrow-\mu_{1}\right)$ to obtain a representation of $\mathcal{R}\left(-\mu_{1} ; x, y\right)$, in 


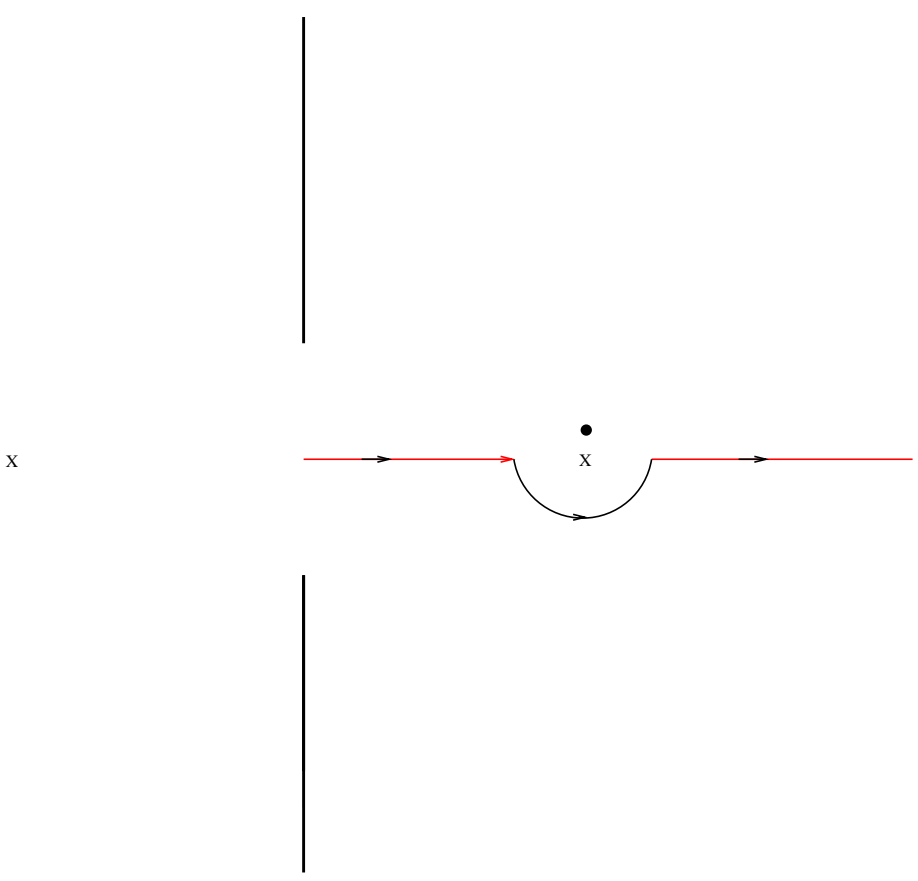

FIG. 5. Complex $\rho$ integration contour for $\mathcal{R}\left(\mu_{1} ; x, y\right)$. The points $\pm \mu^{\prime}$ are denoted by " $x$." The point $\mu_{1}=\mu^{\prime}+i \epsilon_{1}, \epsilon_{1}>0$ is denoted by a circle in the upper half-plane. Vertical semi-infinite lines are branch cuts extending from $\pm i m$ to $\pm i \infty$.

terms of integration over a contour containing $\gamma_{+}$; see Figure 7 . The difference, $\mathcal{R}\left(\mu_{1} ; x, y\right)-\mathcal{R}\left(-\mu_{1} ; x, y\right)$, has an integral representation over the contour homotopic to $\gamma_{-}\left(\mu^{\prime}\right)-\gamma_{+}\left(\mu^{\prime}\right)$, a circle about $\mu^{\prime}$ traversed counterclockwise; see Figure 8. Thus

$$
\begin{aligned}
\mathcal{R}_{j}\left(\mu_{1} ; x, y\right)-\mathcal{R}_{j}\left(-\mu_{1} ; x, y\right) & \\
\quad= & \left(m^{2}+\mu_{1}^{2}\right)^{2}(2 \pi)^{-n} \int_{S^{n-1}} d \omega \oint_{\gamma_{+}-\gamma_{-}} d \rho \rho^{n-1} e^{i \rho \omega \cdot(x-y)} \frac{1}{\left(\rho^{2}-\mu_{1}^{2}\right)\left(m^{2}+\rho^{2}\right)^{2-j / 2}} \\
\quad= & \left(m^{2}+\mu_{1}^{2}\right)^{\frac{j}{2}} \mu_{1}^{n-2}(\pi i)(2 \pi)^{-n} \int_{S^{n-1}} d \omega e^{i \mu_{1} \omega \cdot(x-y)} \\
& =\left(m^{2}+\mu_{1}^{2}\right)^{\frac{j}{2}}(\pi i)(2 \pi)^{\frac{-n}{2}}\left(\frac{\mu_{1}}{|x-y|}\right)^{\frac{n-2}{2}} J_{\frac{n-2}{2}}\left(\mu_{1}|x-y|\right) .
\end{aligned}
$$

In the second equality of (3.25), we have performed the contour integral, and in the third we have used the well-known expression (1.18) for the Fourier transform of the uniform measure on the sphere, $S^{n-1}$, in terms of Bessel functions. This completes the proof of (3.20).

To prove (3.21), we rewrite the kernel $\mathcal{R}_{j}$ as

$$
\begin{aligned}
\mathcal{R}_{j}(\mu ; x, y)= & \left(m^{2}+\mu^{2}\right)^{\frac{j}{2}} \int \frac{d \xi}{(2 \pi)^{n}} e^{i \xi \cdot(x-y)} \frac{1}{\xi^{2}-\mu^{2}} \\
& +\left(m^{2}+\mu^{2}\right)^{2} \int \frac{d \xi}{(2 \pi)^{n}} e^{i \xi \cdot(x-y)}\left(\frac{\left(m^{2}+\xi^{2}\right)^{j / 2-2}-\left(m^{2}+\mu^{2}\right)^{j / 2-2}}{\xi^{2}-\mu^{2}}\right) .
\end{aligned}
$$




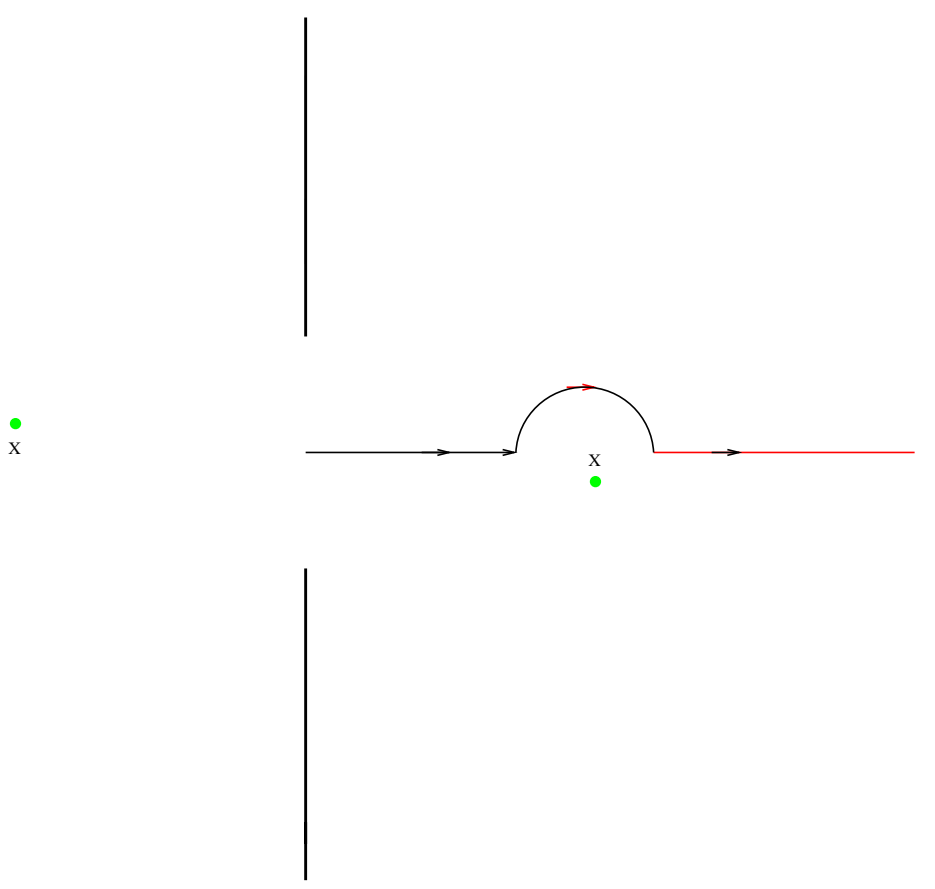

FIG. 6. Integration contour for $\mathcal{R}\left(\mu_{2} ; x, y\right)$. The points $\mu_{2}=-\mu^{\prime}+i \epsilon_{2}, \epsilon_{2}>0$, and $-\mu_{2}$ are denoted by circles.

We recognize the first term in (3.26) as a multiple of a Hankel function, which, for $\Im \mu>0$, by a contour deformation decays exponentially as $|x-y| \rightarrow \infty$. It is therefore proportional to the outgoing Hankel function. The integrand in the second term, along with any number of derivatives with respect to $\mu$, is analytic in $\xi$ in a strip around the real axes and has sufficiently rapid decay as $|\xi| \rightarrow \infty$ so that a Paley-Wiener-type theorem (e.g., Theorem IX.13 of [21]) applies to prove (3.22) for $n=2,3$.

Proof of Lemma 3.5. For $\Im \mu>0$ the boundedness of $T_{R}$ in $L^{2}$ follows from bounding its Fourier transform, which is simple because the resolvent kernel decays exponentially for $\Im \mu>0$. However, the analytic continuation of the resolvent kernel to $\Im \mu<0$ grows exponentially, so we must proceed more carefully. We use a combination of techniques; see, for example, $[2,15]$.

By commuting $\langle D\rangle$, we rewrite $T_{R}$ as a sum of operators, whose kernels can be analytically continued. The proof uses two technical results, Lemmas 3.8 and 3.9, which are stated and proved at the end of this section.

First, consider the case $k=0$ :

$$
\begin{aligned}
T_{R} \equiv & \langle D\rangle \chi R_{0}(\mu) \chi\langle D\rangle \\
= & (\chi\langle D\rangle+[\langle D\rangle, \chi]) R_{0}(\mu)(\langle D\rangle \chi+[\chi,\langle D\rangle]) \\
= & \chi\langle D\rangle R_{0}\langle D\rangle \chi+\left([\langle D\rangle, \chi] \tilde{\chi}^{-1}\right)\left(\tilde{\chi} R_{0}\langle D\rangle \chi\right)+\left(\chi\langle D\rangle R_{0} \tilde{\chi}\right)\left(\tilde{\chi}^{-1}[\chi,\langle D\rangle]\right) \\
& +\left([\langle D\rangle, \chi] \tilde{\chi}^{-1}\right)\left(\tilde{\chi} R_{0} \tilde{\chi}\right)\left(\tilde{\chi}^{-1}[\chi,\langle D\rangle]\right) \\
= & A_{\mathrm{I}}+A_{\mathrm{II}}^{(a)}+A_{\mathrm{II}}^{(b)}+A_{\mathrm{III}} .
\end{aligned}
$$

Then estimate each of the terms in succession.

We begin with $A_{\mathrm{I}}$. For $\mu$ in the upper half-plane, the Fourier representation of 


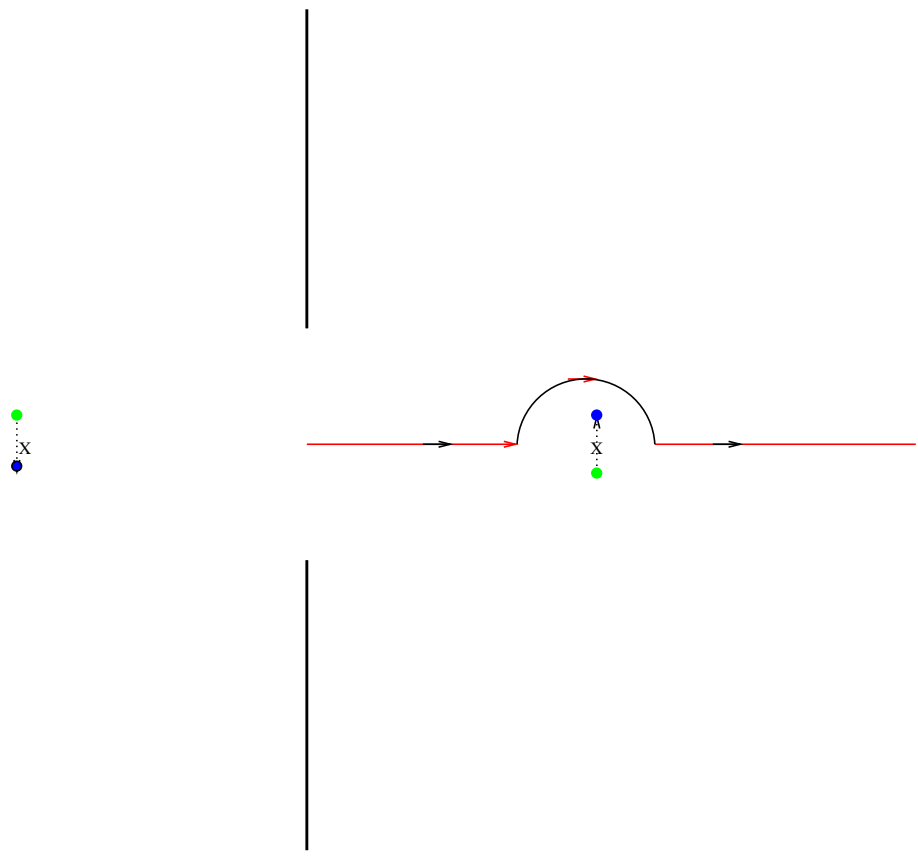

FIG. 7. We let $\epsilon_{2}$ approach $-\epsilon_{1}$, corresponding to $\mu_{2} \rightarrow-\mu_{1}$. Integration contour shown is for $\mathcal{R}\left(-\mu_{1} ; x, y\right)$.

$R_{0}(\mu)$ is valid, and we may therefore commute one of the $\langle D\rangle$ operators through the resolvent and apply the identity

$$
\chi R_{0}(\mu)\langle D\rangle^{2} \chi=\chi I \chi+\left(m^{2}+\mu^{2}\right) \chi R_{0}(\mu) \chi .
$$

The expression (3.28) enables us to carry out the analytic continuation of $A_{\mathrm{I}}$ from the upper half-plane to the lower half-plane. Boundedness on $L^{2}$ in the required region follows from Lemma 3.8 with $k=0$ below and (3.3).

Moving to the next two terms, we observe that it is sufficient to discuss only one of $A_{\mathrm{II}}^{(a)}$ and $A_{\mathrm{II}}^{(b)}$, since $\langle D\rangle$ commutes with $R_{0}$ in the upper half-plane, and the analytic continuation is given by Lemma 3.7. We decompose

$$
A_{\mathrm{II}}^{(a)}=\left([\langle D\rangle, \chi] \tilde{\chi}^{-1}\right)\left(\tilde{\chi} R_{0}\langle D\rangle \chi\right)
$$

and note that the first term is bounded by Lemma 3.9. We show boundedness of the second term in two steps. We first consider $\Im \mu \geq 0$. Then (3.18) and (3.21) allow us to express $R_{0}(\mu)\langle D\rangle$ as a sum of four operators. The two containing Bessel kernels are clearly bounded, while the term containing $R_{0}$ is bounded by Lemma 3.8. That $\mathcal{R}_{j}^{(2)}$ is bounded can be seen by computing the $L^{1} \rightarrow L^{\infty}$ norm of its kernel, using (3.22).

Next, choose $\mu$ such that $\Im \mu>-m$. Then (3.20) allows us to express $R_{0}(\mu)\langle D\rangle$ as a sum of two terms, the first of which, $R_{0}(-\mu)\langle D\rangle$, we have already bounded and the second of which is bounded by Lemma 3.8.

Finally, the last term $A_{\text {III }}$ is bounded by application of Lemmas 3.9 and 3.8.

The result for $k>0$ is obtained by differentiating the kernels $R_{0}$ and $\mathcal{L}_{1}$ in (3.27) 


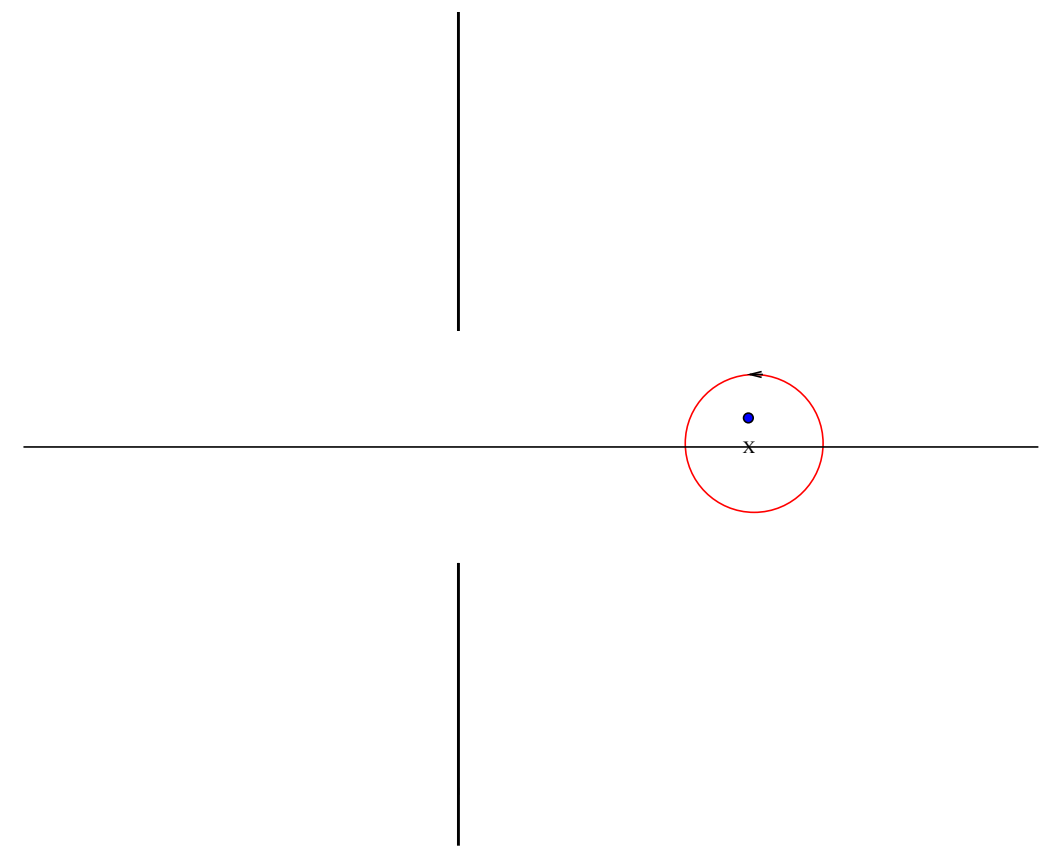

FIG. 8. The jump in $\mathcal{R}(\mu ; x, y)$ from its value at $\mu_{1}$ on the first Riemann sheet to its value on the second Riemann sheet, $\mathcal{R}\left(\mu_{1} ; x, y\right)-\mathcal{R}\left(-\mu_{1} ; x, y\right)$, is represented as an integral over the circular contour shown.

and proceeding as above, noting that Lemmas 3.7 and 3.8 can be applied for any $k>0$.

Lemma 3.8. The operator mapping $L^{2}\left(\mathbb{R}^{n}\right) \rightarrow L^{2}\left(\mathbb{R}^{n}\right)$ given by the kernel

$$
\partial_{\mu}^{k}\left[\chi(x)\left(\frac{\mu}{|x-y|}\right)^{\frac{n-2}{2}} \mathcal{J}_{\frac{n-2}{2}}(\mu|x-y|) \chi(y)\right]
$$

is Hilbert-Schmidt, and therefore compact and, in particular, bounded, for $\Im \mu>-m$ and any $k \geq 0$. In (3.29), $\mathcal{J}$ may be any of the Bessel functions $J, Y, H^{(1)}$, or $H^{(2)}$.

This result, for dimension $n=3$, follows from the proof in [2]; see, in particular, the analysis of the operator denoted $T_{\kappa}$. The case of general $n$ follows the same line of argument.

LEMMA 3.9. Let $\chi$ and $\tilde{\chi}$ be exponentially localized functions with exponential rates $M$ and $\tilde{M}$ as introduced in (1.15). Then, the operator

$$
\mathcal{C}=[\langle D\rangle, \chi] \tilde{\chi}^{-1}: L^{2}\left(\mathbb{R}^{n}\right) \rightarrow L^{2}\left(\mathbb{R}^{n}\right)
$$

is bounded when $\tilde{M}<\min (m, M / \sqrt{2})$. For any $f \in L^{2}\left(\mathbb{R}^{n}\right)$

$$
\overline{\mathcal{C} f}=\mathcal{C} \bar{f} .
$$

The adjoint of $\mathcal{C}, \mathcal{C}^{*}$, is given by

$$
\mathcal{C}^{*}=\tilde{\chi}^{-1}[\chi,\langle D\rangle]
$$


Proof of Lemma 3.9. We first show that $\mathcal{C}$ is bounded. Let $f$ denote any member of $L^{2}\left(\mathbb{R}^{n}\right)$. Then,

$$
\mathcal{C} f \equiv F(x)=\int d y \int \frac{d \zeta}{(2 \pi)^{n}} \frac{d \eta}{(2 \pi)^{n}} e^{i \zeta \cdot x-i \eta \cdot y} \hat{\chi}(\zeta-\eta)(\langle\zeta\rangle-\langle\eta\rangle) \tilde{\chi}^{-1}(y) f(y) .
$$

Since $\|\mathcal{C} f\|_{2}=\|\hat{F}\|_{2}$ it suffices to show that $\|\hat{F}\|_{2}$ is bounded above by $C\|f\|_{2}$ for some positive constant, $C$. The integrand in (3.33) is analytic in $\zeta$ varying over the product of strips around the real axes, $-\infty<\zeta_{j}<\infty$, and has sufficient decay as $\left|\zeta_{j}\right| \rightarrow \infty$ so that we may shift the $\zeta$ contours $\zeta_{j} \rightarrow \zeta_{j}+i \gamma_{j}$, with $|\gamma|<\min (m, M)$. We next write

$$
\tilde{\chi}^{-1}(y)=\sum_{s \in\{-1,1\}^{n}} \mathbf{1}_{\operatorname{sgn}(y)=s}(y) \tilde{\chi}^{-1}(y)
$$

and define $\gamma_{j}=\gamma_{j}(s)=-v s_{j}$, where $v$ is chosen to satisfy

$$
n^{-\frac{1}{2}} \tilde{M}<v<n^{-\frac{1}{2}} \min (m, M) .
$$

We note that the first inequality in (3.34) will ensure that, for $y \in \mathbb{R}^{n}$ and $|y|_{1}=$ $\left|y_{1}\right|+\cdots+\left|y_{n}\right|$,

$$
\left\|\mathbf{1}_{\operatorname{sgn}(y)=s}(y) \tilde{\chi}^{-1}(y) e^{-v|y|_{1}} f(y)\right\|_{2} \leq C\|f\|_{2},
$$

while the second ensures that $|\gamma|<\min (m, M)$, so that the contour shift is permitted.

After the contour shift, we have

$$
\begin{aligned}
\hat{F}(\xi)= & \sum_{s \in\{-1,1\}^{n}} \int d y \int \frac{d \eta}{(2 \pi)^{n}} e^{-i \eta \cdot y} \hat{\chi}(\xi-\eta-i \gamma(s))(\langle\xi\rangle-\langle\eta+i \gamma(s)\rangle) \\
& \times \mathbf{1}_{\operatorname{sgn}(y)=s}(y) \tilde{\chi}^{-1}(y) e^{-v|y|_{1}} f(y) .
\end{aligned}
$$

To continue, we define

$$
g_{s}(y)=\mathbf{1}_{\operatorname{sgn}(y)=s}(y) \tilde{\chi}^{-1}(y) e^{-v|y|_{1}}
$$

and carry out the $d y$ integral:

$$
\hat{F}(\xi)=\sum_{s \in\{-1,1\}^{n}} \int \frac{d \eta}{(2 \pi)^{n}} \underbrace{\hat{\chi}(\xi-\eta-i \gamma)}_{F_{1}} \underbrace{\langle\langle\xi\rangle-\langle\eta+i \gamma\rangle)}_{F_{2}} \hat{g}_{s}(\eta) .
$$

We may bound $F_{1}$ of (3.36) as follows. Choose $\gamma^{2}=(\delta / 2) M^{2}$, with $\delta \in(0,1)$, and set $x=\xi-\eta$. Then

$$
\begin{aligned}
\left|M^{2}+(x-i \gamma)^{2}\right|^{2} & =\left(M^{2}+x^{2}\right)^{2}+\gamma^{4}-2 \gamma^{2}\left(M^{2}+x^{2}\right)+4(x \cdot \gamma)^{2} \\
& \geq\left(M^{2}+x^{2}\right)\left(M^{2}+x^{2}-2 \gamma^{2}\right) \\
& \geq\left((1-\delta) M^{2}+x^{2}\right)^{2}
\end{aligned}
$$

and it follows that

$$
\left|F_{1}\right| \leq\left((1-\delta) M^{2}+(\xi-\eta)^{2}\right)^{-4}
$$


We bound $F_{2}$ of (3.36) as follows. Write $F_{2}=F_{2}^{(1)}+F_{2}^{(2)}$, with

$$
\begin{aligned}
& F_{2}^{(1)}=\left(m^{2}+\xi^{2}\right)^{\frac{1}{2}}-\left(m^{2}+\eta^{2}\right)^{\frac{1}{2}}, \\
& F_{2}^{(2)}=\left(m^{2}+\eta^{2}\right)^{\frac{1}{2}}-\left(m^{2}+(\eta+i \gamma)^{2}\right)^{\frac{1}{2}} .
\end{aligned}
$$

We observe that $\left|F_{2}^{(1)}\right| \leq C|\xi-\eta|$. We may bound $\left|F_{2}^{(2)}\right|$ by noting that it can be written as

$$
F_{2}^{(2)}=|\eta|\left(1+f_{1}(\eta)\right)-|\eta|\left(1+f_{2}(\eta)\right)
$$

where

$$
\left|f_{1}(\eta)\right| \leq O\left(|\eta|^{-2}\right), \quad\left|f_{2}(\eta)\right| \leq O\left(|\eta|^{-1}\right) .
$$

Then, clearly $\left|F_{2}^{(2)}\right|$ is bounded by a constant, and

$$
\left|F_{2}\right| \leq C+|\xi-\eta|
$$

Finally, we assemble (3.38) and (3.39) by writing

$$
|\hat{F}(\xi)| \leq(2 \pi)^{-n} \sum_{s \in\{-1,1\}^{n}}\left(h \star\left|\hat{g}_{s}\right|\right)(\xi)
$$

with

$$
h(\xi-\eta)=\left((1-\delta) M^{2}+(\xi-\eta)^{2}\right)^{-4}(C+|\xi-\eta|) .
$$

Then, by Young's inequality,

$$
\|\mathcal{C} f\|_{2}=\|\hat{F}\|_{2} \leq \pi^{-n}\|h\|_{1} \sup _{s \in\{-1,1\}^{n}}\left\|\hat{g}_{s}\right\|_{2} \leq C\|f\|_{2} .
$$

The expression (3.31) follows from (3.33), as does (3.32) for the adjoint $\mathcal{C}^{*}$, along with the fact that $\mathcal{C}$ is bounded.

4. Perturbation theory of scattering resonances. In this section we state and prove a perturbation theorem for solutions of the scattering resonance problem. We first consider, in section 4.1, the case where the resonance subspace being perturbed has dimension one. We then turn, in section 4.2, to the two-dimensional radial case, $V_{0}=V_{0}(r)$, and show how the perturbation theory of degenerate pairs of resonances (occurring for angular momentum $|l| \geq 1$ ) can be reduced to the previous case.

\subsection{Perturbations of simple scattering resonances.}

TheOrEm 4.1. (a) Let $V_{0}$ denote a potential for which $\left\|V_{0}\right\| \mid$ is finite, where

$$
\left\|\left|V_{0}\|\mid \equiv\| T_{V_{0}}\left\|_{\mathcal{B}\left(L^{2}\right)}=\right\|\langle D\rangle^{-1} V_{0}\langle D\rangle^{-1} \|_{\mathcal{B}\left(L^{2}\right)} .\right.\right.
$$

Let $\left(E_{0}, \psi_{0}\right)$ denote a solution of the corresponding scattering resonance problem. Assume that

$$
\operatorname{dim}\left\{\operatorname{ker}\left(I+T_{R}\left(E_{0}\right) T_{V_{0}}\right)\right\}=1
$$


and that this subspace is spanned by the function

$$
\Psi_{0}=\langle D\rangle \chi \psi_{0}
$$

(see (3.9)). Furthermore, assume the condition

$$
\left\langle T_{V_{0}} \bar{\Psi}_{0}, T_{R}^{\prime}\left(E_{0}\right) T_{V_{0}} \Psi_{0}\right\rangle \neq 0 .
$$

Then, there exists $\varepsilon_{0}$ such that for any potential $V$ satisfying $\left\|V-V_{0}\right\| \mid<\varepsilon_{0}$, there corresponds a unique solution $(E(V), \psi(V))$ of the scattering resonance problem which lies near $\left(E_{0}, \psi_{0}\right)$.

(b) The mapping

$$
V \mapsto(E, \psi) \in \mathbb{C} \times H^{1}(\chi(x) d x),
$$

which associates to a potential $V$ a solution $(E(V), \psi(V))$ of the scattering resonance problem, is analytic in the norm $\|V \mid\|$ in a neighborhood $\left\|V-V_{0}\right\|<\varepsilon_{0}$ of $V_{0}$.

Proof of Theorem 4.1. We formulate the problem so that the result follows from the implicit function theorem. For $\left\|\left|V-V_{0} \|\right|\right.$ small we seek a solution of the scattering resonance problem

$$
\left(I+T_{R}(E) T_{V}\right) \Psi=0
$$

in the form

$$
\begin{aligned}
& E=E_{0}+\delta E, \\
& \Psi=\Psi_{0}+\delta \Psi,
\end{aligned}
$$

where

$$
\left(I+T_{R}\left(E_{0}\right) T_{V_{0}}\right) \Psi_{0}=0 .
$$

Substitution of (4.5) and (4.6) into (4.4) and use of (4.7) yields

$$
\begin{aligned}
\left(I+T_{R}\left(E_{0}\right) T_{V_{0}}\right) \delta \Psi= & -T_{R}\left(E_{0}\right) T_{\delta V} \Psi_{0}-\delta T_{R}\left(E_{0}, \delta E\right) T_{V_{0}} \Psi_{0} \\
& -\mathcal{Q}\left(\delta V, \delta \Psi, \delta E ; V_{0}, \Psi_{0}, E_{0}\right)
\end{aligned}
$$

with

$$
\delta T_{R}\left(E_{0}, \delta E\right)=T_{R}\left(E_{0}+\delta E\right)-T_{R}\left(E_{0}\right)
$$

and $\mathcal{Q}$ consisting only of the quadratic and higher order terms:

$$
\begin{aligned}
\mathcal{Q}\left(\delta V, \delta \Psi, \delta E ; V_{0}, \Psi_{0}, E_{0}\right)= & \left(T_{R}\left(E_{0}\right) T_{\delta V}+\delta T_{R}\left(E_{0}, \delta E\right) T_{V_{0}}\right) \delta \Psi \\
& +\delta T_{R}\left(E_{0}, \delta E\right) T_{\delta V}\left(\Psi_{0}+\delta \Psi\right) .
\end{aligned}
$$

We will apply the analytic Fredholm theorem to (4.8). The solvability condition requires an understanding of the adjoint operator $I+T_{V_{0}}^{*} T_{R}\left(E_{0}\right)^{*}$. Note that since $V_{0}$ is real-valued, $T_{V_{0}}$ is self-adjoint.

LEMma 4.2. Under condition (4.2) of Theorem 4.1, the adjoint operator I+ $T_{V_{0}} T_{R}\left(E_{0}\right)^{*}$ has a one-dimensional null space spanned by the function

$$
\Psi_{0}^{\#}=T_{V_{0}} \bar{\Psi}_{0} .
$$


Therefore, the inhomogeneous problem

$$
\left(I+T_{R}\left(E_{0}\right) T_{V_{0}}\right) U=S
$$

has a solution for $S \in L^{2}$ if and only if

$$
\left\langle T_{V_{0}} \bar{\Psi}_{0}, S\right\rangle=0
$$

Proof of Lemma 4.2. Since $T_{R}\left(E_{0}\right) T_{V_{0}}$ is compact the dimensions of the null spaces of $I+T_{R}\left(E_{0}\right) T_{V_{0}}$ and $I+T_{V_{0}} T_{R}\left(E_{0}\right)^{*}$ are equal [12].

To construct the adjoint null space, observe that an element of the null space satisfies

$$
\left(I+T_{R}\left(E_{0}\right) T_{V_{0}}\right) \Psi_{0}=0
$$

Applying the operator $T_{V_{0}}$ we get

$$
\left(I+T_{V_{0}} T_{R}\left(E_{0}\right)\right) T_{V_{0}} \Psi_{0}=0 .
$$

Taking the complex conjugate of (4.13) yields

$$
\overline{T_{V_{0}} \Psi_{0}}+\overline{T_{V_{0}} T_{R}\left(E_{0}\right) T_{V_{0}} \Psi_{0}}=0 .
$$

The lemma follows from the observation that $\overline{T_{V_{0}} f}=T_{V_{0}} \bar{f}$, since $V_{0}$ is real-valued, and the conclusion of Lemma 4.3.

Lemma 4.3. For any $\mu \in \mathbb{C} \backslash\{0\}$ satisfying $\Im \mu>-\tilde{M}>-m$ and $f \in L^{2}\left(\mathbb{R}^{n}\right)$,

$$
\overline{T_{R}(\mu) f}=T_{R}(\mu)^{*} \bar{f} .
$$

Proof of Lemma 4.3. We employ the decomposition (3.27) of $T_{R}$. We first note that the result for $A_{\mathrm{I}}$ follows from the use of the identity (3.28), Lemma 3.8, and the fact that the free resolvent is symmetric, as can be seen from (3.3). We next consider

$$
A_{\mathrm{II}}=A_{\mathrm{II}}^{(a)}+A_{\mathrm{II}}^{(b)}=\mathcal{C}\left(\tilde{\chi} \mathcal{L}_{1}(\mu) \chi\right)+\left(\chi \mathcal{L}_{1}(\mu) \tilde{\chi}\right) \mathcal{C}^{*},
$$

where we have used the notation of (3.30) and (3.17). The result follows from Lemma 3.9, which tells us that $\overline{\mathcal{C} f}=\mathcal{C} \bar{f}$ for $f \in L^{2}$; Lemma 3.7, which tells us that $\mathcal{L}_{1}$ has a symmetric kernel; and Lemma 3.8, which tells us that $\tilde{\chi} \mathcal{L}_{1}(\mu) \chi$ is a bounded operator from $L^{2} \rightarrow L^{2}$ for $\Im \mu>-\tilde{M}$. The result for $A_{\text {III }}$ follows similarly.

Continuing with the proof of Theorem 4.1, we note that (4.11) gives us an implicit condition for the solvability of (4.8) for $\delta \Psi$, obtained by setting the inner product of the right-hand side of (4.8) with $T_{V_{0}} \bar{\Psi}_{0}$ equal to zero:

$$
\begin{array}{r}
\left\langle T_{V_{0}} \bar{\Psi}_{0}, \delta T_{R}\left(E_{0}, \delta E\right) T_{V_{0}} \Psi_{0}\right\rangle+\left\langle T_{V_{0}} \bar{\Psi}_{0}, T_{\delta V} \Psi_{0}\right\rangle \\
\quad+\left\langle T_{V_{0}} \bar{\Psi}_{0}, \mathcal{Q}\left(\delta V, \delta \Psi, \delta E ; V_{0}, \Psi_{0}, E_{0}\right)\right\rangle=0 .
\end{array}
$$

We now view the task of solving the scattering resonance problem as that of seeking a solution $(\Psi(V), E(V))$ of the system of equations (4.8), (4.15). In compact form we write

$$
\mathbf{F}(\delta V, \delta \Psi, \delta E)=\mathbf{0}
$$


where $\mathbf{F}=\left(F_{1}, F_{2}\right)$ and

$$
\begin{aligned}
F_{1}(\delta V, \delta \Psi, \delta E)= & \left(I+T_{R}\left(E_{0}\right) T_{V_{0}}\right) \delta \Psi+\left(T_{R}\left(E_{0}\right) T_{\delta V}+\delta T_{R}\left(E_{0}, \delta E\right) T_{V_{0}}\right) \Psi_{0} \\
& +\mathcal{Q}\left(\delta V, \delta \Psi, \delta E ; V_{0}, \Psi_{0}, E_{0}\right) \\
F_{2}(\delta V, \delta \Psi, \delta E,)= & \left\langle T_{V_{0}} \bar{\Psi}_{0},\left(T_{R}\left(E_{0}\right) T_{\delta V}+\delta T_{R}\left(E_{0}, \delta E\right) T_{V_{0}}\right) \Psi_{0}\right. \\
& \left.+\mathcal{Q}\left(\delta V, \delta \Psi, \delta E ; V_{0}, \Psi_{0}, E_{0}\right)\right\rangle
\end{aligned}
$$

We verify that the hypotheses of the analytic implicit function theorem hold for $\mathbf{F}: \mathbf{X} \times \mathbf{Y} \rightarrow \mathbf{Z}$, with

$$
\begin{aligned}
& \mathbf{X}=\overline{\left\{V \in L^{2}\left(\mathbb{R}^{n}\right) \text { of compact support }\right\}} \\
& \mathbf{Y}=L^{2}\left(\mathbb{R}^{n}\right) \times \mathbb{C} \\
& \mathbf{Z}=\left\{(f, z) \in L^{2}\left(\mathbb{R}^{n}\right) \times \mathbb{C}: z=\left\langle T_{V_{0}} \bar{\Psi}_{0}, f\right\rangle\right\}
\end{aligned}
$$

and with the norms $\|V\| \|$ for $V \in \mathbf{X},\|\Psi\|_{L^{2}}+|E|$ for $(\Psi, E) \in \mathbf{Y}$, and $\|f\|_{L^{2}}+|z|$ for $(f, z) \in \mathbf{Z}$.

We first compute the differential $D \mathbf{F}$ evaluated at a point $x_{0}=(\tilde{V}, \tilde{\Psi}, \tilde{E})$ in a neighborhood of the origin:

$$
\begin{aligned}
& D_{\delta V, \delta \Psi, \delta E} F_{1}\left(x_{0}\right)=D_{\delta V} F_{1}\left(x_{0}\right)+D_{\delta \Psi} F_{1}\left(x_{0}\right)+D_{\delta E} F_{1}\left(x_{0}\right) \\
& =T_{R}\left(E_{0}\right) T_{\delta V} \Psi_{0}+T_{R}\left(E_{0}\right) T_{\delta V} \tilde{\Psi}+\delta T_{R}\left(E_{0}, \tilde{E}\right) T_{\delta V}\left(\Psi_{0}+\tilde{\Psi}\right) \\
& +\left[I+T_{R}\left(E_{0}\right) T_{V_{0}}+T_{R}\left(E_{0}\right) T_{\tilde{V}}+\delta T_{R}\left(E_{0}, \tilde{E}\right)+\delta T_{R}\left(E_{0}, \tilde{E}\right) T_{\tilde{V}}\right] \delta \Psi \\
& +T_{R}^{\prime}\left(E_{0}+\tilde{E}\right)\left[T_{V_{0}} \Psi_{0}+T_{V_{0}} \tilde{\Psi}+T_{\tilde{V}}\left(\Psi_{0}+\tilde{\Psi}\right)\right] \delta E, \\
& D_{\delta V, \delta \Psi, \delta E} F_{2}\left(x_{0}\right)=D_{\delta V} F_{2}\left(x_{0}\right)+D_{\delta \Psi} F_{2}\left(x_{0}\right)+D_{\delta E} F_{2}\left(x_{0}\right) \\
& =\left\langle T_{V_{0}} \bar{\Psi}_{0}, T_{R}\left(E_{0}\right) T_{\delta V} \Psi_{0}+T_{R}\left(E_{0}\right) T_{\delta V} \tilde{\Psi}+\delta T_{R}\left(E_{0}, \tilde{E}\right) T_{\delta V}\left(\Psi_{0}+\tilde{\Psi}\right)\right. \\
& +\left[T_{R}\left(E_{0}\right) T_{\tilde{V}}+\delta T_{R}\left(E_{0}, \tilde{E}\right)+\delta T_{R}\left(E_{0}, \tilde{E}\right) T_{\tilde{V}}\right] \delta \Psi \\
& \left.+T_{R}^{\prime}\left(E_{0}+\tilde{E}\right)\left[T_{V_{0}} \Psi_{0}+T_{V_{0}} \tilde{\Psi}+T_{\tilde{V}}\left(\Psi_{0}+\tilde{\Psi}\right)\right] \delta E\right\rangle .
\end{aligned}
$$

By Lemmas 3.4 and 3.5, we see that

$$
\left\|D_{\delta V, \delta \Psi, \delta E} \mathbf{F}\left(x_{0}\right)\right\|_{\mathbf{z}} \leq C\|\| \delta V\||+C\|\delta \Psi\|+C| \delta E \mid
$$

for $x_{0}$ in a neighborhood of the origin, and therefore $\mathbf{F}$ is analytic there. We observe that $\mathbf{F}(0,0,0)=0$ and consider the differential evaluated at the origin:

$$
D_{\delta \Psi, \delta E} \mathbf{F}(0,0,0)=\left(\begin{array}{cc}
I+T_{R}\left(E_{0}\right) T_{V_{0}} & T_{R}^{\prime}\left(E_{0}\right) T_{V_{0}} \Psi_{0} \\
0 & \left\langle T_{V_{0}} \Psi_{0}, T_{R}^{\prime}\left(E_{0}\right) T_{V_{0}} \Psi_{0}\right\rangle
\end{array}\right) .
$$

We now verify that the inverse of $D_{\delta \Psi, \delta E} \mathbf{F}(0,0,0)$ is defined and bounded on $\mathbf{Z}$. Consider the system of equations

$$
\begin{aligned}
\left(I+T_{R}\left(E_{0}\right) T_{V_{0}}\right) \delta \Psi+T_{R}^{\prime}\left(E_{0}\right) T_{V_{0}} \bar{\Psi}_{0} \delta E & =f_{1}, \\
\left\langle T_{V_{0}} \bar{\Psi}_{0}, T_{R}^{\prime}\left(E_{0}\right) T_{V_{0}} \Psi_{0}\right\rangle E & =\left\langle T_{V_{0}} \bar{\Psi}_{0}, f_{1}\right\rangle .
\end{aligned}
$$

By Lemma 4.2 , this system can be solved uniquely for $(\delta \Psi, \delta E) \in L^{2} \times \mathbb{C}$ of a function of $\delta V$. The conclusions of Theorem 4.1 now follow from the implicit function theorem. 
4.2. Perturbation theory of degenerate resonances: A special case. There are many situations in which degenerate resonances occur or, equivalently, cases where assumption (4.2) is violated. A general theory of degenerate scattering resonances will not be developed in this work. We focus instead on a particular class of potentials having degenerate resonances and their behavior under a class of perturbations.

In particular, we consider the case of potentials in the plane with radial symmetry, $V_{0}(r)$, and rapidly varying, zero mean, perturbations of the type illustrated in Figure 1.2, $\delta V(r, N \theta)$. Assumption (4.2) on the resonances of the unperturbed potential is violated because of the presence of a double degeneracy indexed by positive and negative angular momenta. In this section we prove that for this class of perturbations, the perturbation theory of a twofold degenerate resonance reduces to that of two independent nondegenerate resonances, as formally seen in [7].

We begin by noting that solutions of the unperturbed problem

$$
\left(I+T_{R}\left(E_{0}\right) T_{V_{0}}\right) \Psi_{0}=0
$$

divide into degenerate subspaces of angular momentum $\ell$ spanned by

$$
\Psi_{0 \pm \ell}=e^{ \pm i \ell \theta} \tilde{\Psi}_{0 \ell}
$$

Here, $\tilde{\Psi}_{0 \ell}=\tilde{\Psi}_{0 \ell}(r)$ satisfies

$$
\left(I+\check{T}_{\ell, R}\left(E_{0}\right) \check{T}_{\ell, V_{0}}\right) \tilde{\Psi}_{0 \ell}=0
$$

and we have defined

$$
\begin{aligned}
\check{T}_{\ell, R}(E) & =\left\langle\check{D}_{\ell}\right\rangle \chi \check{G}_{\ell}(E) \chi\left\langle\check{D}_{\ell}\right\rangle, \\
\check{T}_{\ell, V} & =\left\langle\check{D}_{\ell}\right\rangle^{-1} \chi^{-1} V \chi^{-1}\left\langle\check{D}_{\ell}\right\rangle^{-1} .
\end{aligned}
$$

The operators $\check{G}_{\ell}$ and $\left\langle\check{D}_{\ell}\right\rangle$ are defined as

$$
\begin{aligned}
\check{G}_{\ell}(E) & =\left(-\Delta+\frac{\ell^{2}}{r^{2}}-E\right)^{-1}, \\
\left\langle\check{D}_{\ell}\right\rangle^{-1} & =\left(m^{2} I-\Delta+\frac{\ell^{2}}{r^{2}}\right)^{-\frac{1}{2}},
\end{aligned}
$$

and they arise as a result of the commutation formulas

$$
\begin{aligned}
T_{V} e^{i \ell \theta} f=e^{i \ell \theta} \check{T}_{\ell, V} f, \\
T_{R} e^{i \ell \theta} g=e^{i \ell \theta} \check{T}_{\ell, R} g .
\end{aligned}
$$

We note that the operators (4.21) differ from the partial wave Green's function $G_{\ell}$ and smoothing operator $\left\langle D_{\ell}\right\rangle^{-1}$ defined in sections 8 and 6 , in that (4.21) contains a full Laplacian.

We expand $\Psi$, a solution of the scattering resonance problem

$$
\left(I+T_{R}(E) T_{V}\right) \Psi=0
$$

with $V$ near $V_{0}$ and $E$ near the degenerate resonance energy $E_{0}$, in the form

$$
\begin{aligned}
\Psi & =\sum_{\alpha \in\{ \pm \ell\}} c_{\alpha} \Psi_{0 \alpha}+\Phi, \\
E & =E_{0}+\delta E \\
V & =V_{0}+\delta V
\end{aligned}
$$


where $c_{\alpha}$ are constants. Substitution of (4.24) into (4.23) yields the following equation for $\Phi$ :

$$
\left(I+T_{R}\left(E_{0}\right) T_{V_{0}}\right) \Phi=\left(T_{R}\left(E_{0}\right) T_{V_{0}}-T_{R}(E) T_{V}\right)\left(\sum_{\alpha \in\{ \pm \ell\}} c_{\alpha} \Psi_{0 \alpha}+\Phi\right) .
$$

The solvability condition is

$$
\left\langle\Psi_{0 \beta}^{\#},\left(T_{R}\left(E_{0}\right) T_{V_{0}}-T_{R}(E) T_{V}\right)\left(\sum_{\alpha \in\{ \pm \ell\}} c_{\alpha} \Psi_{0 \alpha}+\Phi\right)\right\rangle=0, \quad \beta \in\{ \pm \ell\},
$$

where $\Psi_{0 \beta}^{\#}$ span the adjoint null space of $I+T_{R}\left(E_{0}\right) T_{V_{0}}$. These functions are characterized by the following lemma.

LEMma 4.4. If the null space of $I+T_{R}\left(E_{0}\right) T_{V_{0}}$ is spanned by $\Psi_{0 \pm \ell}$, then the adjoint null space is spanned by

$$
\Psi_{0 \pm \ell}^{\#}=e^{ \pm i \ell \theta} \check{T}_{\ell, V_{0}} \overline{\tilde{\Psi}}_{0 \ell}
$$

Proof of Lemma 4.4. Since $T_{R}\left(E_{0}\right) T_{V_{0}}$ is compact the dimensions of the null spaces of $I+T_{R}\left(E_{0}\right) T_{V_{0}}$ and $I+T_{V_{0}} T_{R}\left(E_{0}\right)^{*}$ are equal [12]. By the same argument as in Lemma 4.2, the functions (4.27) are elements of the adjoint null space. When $\ell>0$ the two functions $\Psi_{0 \pm \ell}^{\#}$ are linearly independent.

Returning to (4.25), we choose to represent the solution $\Phi$ as

$$
\Phi=\sum_{\alpha \in\{ \pm \ell\}} c_{\alpha} e^{i \alpha \theta} \tilde{\Phi}_{\ell}
$$

where we define $\tilde{\Phi}_{\ell}$ to satisfy

$$
\begin{aligned}
(I+ & \left.\check{T}_{\ell, R}\left(E_{0}\right) \check{T}_{\ell, V_{0}}\right) \tilde{\Phi}_{\ell} \\
& =\left(\check{T}_{\ell, R}\left(E_{0}\right) \check{T}_{\ell, V_{0}}-\check{T}_{\ell, R}(E) \check{T}_{\ell, V_{0}}-\check{T}_{\ell, R}(E) \check{T}_{\ell, \delta V}\right)\left(\tilde{\Psi}_{0 \ell}+\tilde{\Phi}_{\ell}\right) .
\end{aligned}
$$

To justify the representation (4.28), we note that the linear combination (4.28) satisfies (4.25), and it is unique by Fredholm theory. The solvability condition (4.26) becomes, with the definition (4.28), the matrix equation

$$
M_{\beta \alpha} c_{\alpha}=0,
$$

where

$$
\begin{aligned}
M_{\beta \alpha}= & \underbrace{\left\langle\Psi_{0 \beta}^{\#},\left(T_{R}\left(E_{0}\right)-T_{R}(E)\right) T_{V_{0}} \Psi_{0 \alpha}\right\rangle}_{M_{\beta \alpha}^{(\mathrm{I})}}-\underbrace{\left\langle\Psi_{0 \beta}^{\#}, T_{R}(E) T_{\delta V} \Psi_{0 \alpha}\right\rangle}_{M_{\beta \alpha}^{(\mathrm{III})}} \\
& +\underbrace{\left\langle\Psi_{0 \beta}^{\#},\left(T_{R}\left(E_{0}\right)-T_{R}(E)\right) T_{V_{0}} \Phi_{\alpha}\right\rangle}_{M_{\beta \alpha}^{\text {(IV) }}}-\underbrace{\left\langle\Psi_{0 \beta}^{\#}, T_{R}(E) T_{\delta V} \Phi_{\alpha}\right\rangle}_{\text {talkes }} .
\end{aligned}
$$

Lemma 4.5. When $N>2 \ell$, the matrix $M$ is diagonal and takes the form

$$
\begin{aligned}
M_{\beta \alpha}= & 2 \pi \delta_{\beta \alpha}\left\langle\check{T}_{\ell, V_{0}} \overline{\tilde{\Psi}}_{0 \ell},\left(\check{T}_{\ell, R}\left(E_{0}\right)-\check{T}_{\ell, R}(E)\right) \check{T}_{\ell, V_{0}} \tilde{\Psi}_{0 \ell}\right\rangle_{\mathrm{rad}} \\
& +2 \pi \delta_{\beta \alpha}\left\langle\check{T}_{\ell, V_{0}} \overline{\tilde{\Psi}}_{0 \ell},\left(\check{T}_{\ell, R}\left(E_{0}\right)-\check{T}_{\ell, R}(E)\right) \check{T}_{\ell, V_{0}} \tilde{\Phi}_{0 \ell}\right\rangle_{\mathrm{rad}} \\
& -2 \pi \delta_{\beta \alpha} \sum_{k \in \mathbb{Z} \backslash\{0\}}\left\langle\check{T}_{\ell, V_{0}} \overline{\tilde{\Psi}}_{0 \ell}, \check{T}_{\ell, R}(E)\left\langle\check{D}_{\alpha}\right\rangle^{-1} \chi^{-1} \delta V_{k} \chi^{-1}\left\langle\check{D}_{\alpha-k N}\right\rangle^{-1} \tilde{\Phi}_{\ell,-k}\right\rangle_{\mathrm{rad}}
\end{aligned}
$$


Proof of Lemma 4.5. We explicitly compute the matrix elements of $M$, starting with

$$
M_{\beta \alpha}^{(\mathrm{I})}=2 \pi \delta_{\beta \alpha}\left\langle\check{T}_{\ell, V_{0}} \overline{\tilde{\Psi}}_{0 \ell},\left(\check{T}_{\ell, R}\left(E_{0}\right)-\check{T}_{\ell, R}(E)\right) \check{T}_{\ell, V_{0}} \tilde{\Psi}_{0 \ell}\right\rangle_{\mathrm{rad}},
$$

which follows from the definitions the commutation formulas (4.22). To compute $M^{(\mathrm{II})}$, we expand $\delta V$ in the Fourier series

$$
\delta V=\sum_{k \neq 0} \delta V_{k}(r) e^{i k N \theta},
$$

which yields

$$
\begin{aligned}
M_{\beta \alpha}^{(\mathrm{II})} & =\sum_{k \neq 0}\left\langle T_{V_{0}} e^{i \beta \theta} \overline{\tilde{\Psi}}_{0 \ell}, T_{R}(E) T_{\delta V_{k} e^{i k N \theta}} e^{i \alpha \theta} \tilde{\Psi}_{0 \ell}\right\rangle \\
& =\sum_{k \neq 0} \int_{0}^{2 \pi} e^{i(\alpha-\beta+k N)} d \theta\left\langle\check{T}_{\ell, V_{0}} \overline{\tilde{\Psi}}_{0 \ell}, \check{T}_{\alpha+k N, R}(E)\left\langle\check{D}_{\alpha+k N}\right\rangle^{-1} \chi^{-1} \delta V_{k} \chi^{-1}\left\langle\check{D}_{\ell}\right\rangle \tilde{\Psi}_{0 \ell}\right\rangle \\
& =0 \text { for } N>2 \ell .
\end{aligned}
$$

We see that $M^{(\mathrm{II})}$ vanishes for $N$ large enough. Continuing with $M^{(\mathrm{III})}$, we use the Fourier series of $\tilde{\Phi}_{\ell}$

$$
\tilde{\Phi}_{\ell}(r, \theta)=\sum_{p \in \mathbb{Z}} e^{i N p \theta} \tilde{\Phi}_{\ell, k}(r)
$$

After commuting through the angular dependence, we find

$$
\begin{aligned}
M_{\beta \alpha}^{(\mathrm{III})}=\sum_{p \in \mathbb{Z}} & \int_{0}^{2 \pi} e^{i(\alpha-\beta+p N) \theta} d \theta \\
& \left\langle\check{T}_{\ell, V_{0}} \overline{\tilde{\Psi}}_{0 \ell},\left(\check{T}_{\alpha+p N, R}\left(E_{0}\right)-\check{T}_{\alpha+p N, R}(E)\right) T_{\alpha+p N, V_{0}} \tilde{\Phi}_{\ell, k}\right\rangle_{\mathrm{rad}} .
\end{aligned}
$$

When $\alpha \neq \beta$ and $N>2 \ell, M^{(\mathrm{III})}$ vanishes, but there is a contribution to the diagonal, which comes from the $p=0$ term:

$$
M_{\beta \alpha}^{(\mathrm{III})}=2 \pi \delta_{\beta \alpha}\left\langle\check{T}_{\ell, V_{0}} \overline{\tilde{\Psi}}_{0 \ell},\left(\check{T}_{\ell, R}\left(E_{0}\right)-\check{T}_{\ell, R}(E)\right) \check{T}_{\ell, V_{0}} \tilde{\Phi}_{0 \ell}\right\rangle_{\mathrm{rad}}
$$

Finally, to compute $M^{(\mathrm{IV})}$ we make use of both Fourier series (4.30) and (4.31):

$$
\begin{aligned}
M_{\beta \alpha}^{(\mathrm{IV})}= & \sum_{\substack{k \in \mathbb{Z} \backslash\{0\} \\
p \in \mathbb{Z}}} \int_{0}^{2 \pi} e^{i(\alpha-\beta+k N+p N) \theta} d \theta \\
& \left\langle\check{T}_{\ell, V_{0}} \overline{\tilde{\Psi}}_{0 \ell}, \check{T}_{\alpha+k N+p N, R}(E)\left\langle\check{D}_{\alpha+k N+p N}\right\rangle^{-1} \chi^{-1} \delta V_{k} \chi^{-1}\left\langle\check{D}_{\alpha+p N}\right\rangle^{-1} \tilde{\Phi}_{\ell, p}\right\rangle_{\mathrm{rad}} .
\end{aligned}
$$

Once again, there is no contribution from the off-diagonal terms when $N>2 \ell$, and the diagonal contribution arises from the $k=-p$ terms.

Because $M$ is diagonal for large enough $N$,

$$
M_{\beta \alpha}=\delta_{\beta \alpha} \mathcal{M}_{\beta}(\delta V ; \Phi, \delta E),
$$

the two angular momenta decouple. Choosing $c_{\ell}=1, c_{-\ell}=0$, say, we find that the system (4.25) and (4.26) reduces to (4.29) and $\mathcal{M}_{\ell}(\delta V ; \Phi, d E)=0$. This system is of the same form as that considered in Theorem 4.1, and an analogous result applies. In the current context, the condition (4.3) becomes

$$
\left\langle\check{T}_{\ell, V_{0}} \overline{\tilde{\Psi}}_{0 \ell}, \check{T}_{\ell, R}^{\prime}\left(E_{0}\right) \check{T}_{\ell, V_{0}} \tilde{\Psi}_{0 \ell}\right\rangle_{\mathrm{rad}} \neq 0
$$


5. Scattering resonance expansion. In the previous section we proved that if the scattering resonance problem has a solution $\left(E_{0}, \psi_{0}\right)$ corresponding to a potential $V_{0}$, then for all potentials $V=V_{0}+\delta V$ in a neighborhood of $V_{0}(\|\delta V\| \mid$ small) the scattering resonance problem has a solution $\left(E\left(V_{0}+\delta V\right), \psi\left(V_{0}+\delta V\right)\right)$. Moreover, we have that $E(V)$ and $\psi(V)$ can be expanded about the $V_{0}$ case. In this section we compute the first few terms of this expansion.

To find the explicit expansions of $E(V)$ and $\psi(V)$ we write

$$
\begin{aligned}
& E=E_{0}+\delta E^{(1)}+\delta E^{(2)}+\cdots, \\
& \Psi=\Psi_{0}+\delta \Psi^{(1)}+\delta \Psi^{(2)}+\cdots,
\end{aligned}
$$

where terms with a superscript $j$ are formally of order $j$. Substitution of (5.1) into (4.8) and equating like orders or magnitude yields a hierarchy of inhomogeneous equations, the first two terms of which are

$$
\begin{aligned}
\mathcal{O}(1):\left(I+T_{R}\left(E_{0}\right) T_{V_{0}}\right) \delta \Psi^{(1)}= & -\left(T_{R}\left(E_{0}\right) T_{\delta V}+\delta E^{(1)} T_{R}^{\prime}\left(E_{0}\right) T_{V_{0}}\right) \Psi_{0} \\
\mathcal{O}(2):\left(I+T_{R}\left(E_{0}\right) T_{V_{0}}\right) \delta \Psi^{(2)}= & -\left(\delta E^{(2)} T_{R}^{\prime}\left(E_{0}\right)+\frac{1}{2} T_{R}^{\prime \prime}\left(E_{0}\right)\left(\delta E^{(1)}\right)^{2}\right) T_{V_{0}} \Psi_{0} \\
& -\left(T_{R}\left(E_{0}\right) T_{\delta V}+\delta E^{(1)} T_{R}^{\prime}\left(E_{0}\right) T_{V_{0}}\right) \delta \Psi^{(1)} \\
& -\delta E^{(1)} T_{R}^{\prime}\left(E_{0}\right) T_{\delta V} \Psi_{0} .
\end{aligned}
$$

At order $\mathcal{O}(m)$ in perturbation theory, $\delta E^{(m)}$ is determined by the solvability condition:

$$
\left\langle T_{V_{0}} \bar{\Psi}_{0} \text {, right-hand side of } \delta \Psi^{(m)} \text { equation }\right\rangle=0 .
$$

The equation for $\delta E^{(m)}$ has the form

$$
\left\langle T_{V_{0}} \bar{\Psi}_{0}, T_{R}^{\prime}\left(E_{0}\right) T_{V_{0}} \Psi_{0}\right\rangle \delta E^{(m)}=\cdots .
$$

Therefore, the determination of $\delta E^{(m)}$ at all orders depends on the nonvanishing of the ( $m$-independent) coefficient

$$
C_{d E} \equiv\left\langle T_{V_{0}} \bar{\Psi}_{0}, T_{R}^{\prime}\left(E_{0}\right) T_{V_{0}} \Psi_{0}\right\rangle=\left\langle V_{0} \bar{\psi}_{0}, R_{0}^{\prime}\left(E_{0}\right) V_{0} \psi_{0}\right\rangle .
$$

Consider the $\mathcal{O}(1)$ equation. By Lemma 4.2 a necessary and sufficient condition for solvability in $L^{2}$ is

$$
\left\langle T_{V_{0}} \bar{\Psi}_{0}, T_{R}\left(E_{0}\right) T_{\delta V} \Psi_{0}+\delta E^{(1)} T_{R}^{\prime}\left(E_{0}\right) T_{V_{0}} \Psi_{0}\right\rangle=0
$$

Therefore,

$$
\delta E^{(1)}=-\frac{\left\langle T_{V_{0}} \bar{\Psi}_{0}, T_{R}\left(E_{0}\right) T_{\delta V} \Psi_{0}\right\rangle}{\left\langle T_{V_{0}} \bar{\Psi}_{0}, T_{R}^{\prime}\left(E_{0}\right) T_{V_{0}} \Psi_{0}\right\rangle}
$$

or, equivalently, using (5.6) and the definitions of $T_{V}, T_{R}$, and $\Psi_{0}$,

$$
\delta E^{(1)}=-\frac{\left\langle V_{0} \bar{\psi}_{0}, \delta V \psi_{0}\right\rangle}{\left\langle V_{0} \bar{\psi}_{0}, R_{0}^{\prime}\left(E_{0}\right) V_{0} \psi_{0}\right\rangle} .
$$


If $\delta E^{(1)}$ is chosen to satisfy (5.8), then (5.2) has a unique solution $\Psi^{(1)}$.

Turning to the $\mathcal{O}(2)$ equation, we then substitute this into the right-hand side of (5.3) and find, via Lemma 4.2 , that $\delta E^{(2)}$ is determined by the solvability condition

$$
\begin{aligned}
C_{d E} \delta E^{(2)}= & -\frac{1}{2}\left(\delta E^{(1)}\right)^{2}\left\langle T_{V_{0}} \bar{\Psi}_{0}, T_{R}^{\prime \prime}\left(E_{0}\right) T_{V_{0}} \Psi_{0}\right\rangle \\
& -\left\langle T_{V_{0}} \bar{\Psi}_{0}, T_{R}\left(E_{0}\right) T_{\delta V} \delta \Psi^{(1)}\right\rangle-\delta E^{(1)}\left\langle T_{V_{0}} \bar{\Psi}_{0}, T_{R}^{\prime}\left(E_{0}\right) T_{V_{0}} \delta \Psi^{(1)}\right\rangle \\
& -\delta E^{(1)}\left\langle T_{V_{0}} \bar{\Psi}_{0}, T_{R}^{\prime}\left(E_{0}\right) T_{\delta V} \Psi_{0}\right\rangle .
\end{aligned}
$$

The procedure can be continued to obtain a solution to any order.

Special case: Vanishing first order correction; $\boldsymbol{\delta} \boldsymbol{E}^{(1)}=\mathbf{0}$. When $\delta E^{(1)}=$ 0 , the expression for $\delta E^{(2)}$ simplifies considerably. This case arises in the application of section 6 .

Lemma 5.1. Suppose $\delta E^{(1)}=0$. Then,

$$
\begin{aligned}
\delta E^{(2)} & =C_{d E}^{-1}\left\langle\bar{\Psi}_{0}, T_{\delta V} \delta \Psi^{(1)}\right\rangle \\
& =-\frac{\left\langle T_{\delta V} \bar{\Psi}_{0},\left(I+T_{R}\left(E_{0}\right) T_{V_{0}}\right)^{-1} T_{R}\left(E_{0}\right) T_{\delta V} \Psi_{0}\right\rangle}{\left\langle T_{V_{0}} \bar{\Psi}_{0}, T_{R}^{\prime}\left(E_{0}\right) T_{V_{0}} \Psi_{0}\right\rangle} .
\end{aligned}
$$

Proof of Lemma 5.1. If $\delta E^{(1)}=0$, then (5.9) gives

$$
\left\langle T_{V_{0}} \bar{\Psi}_{0}, \delta E^{(2)} T_{+}^{\prime}\left(E_{0}\right) T_{V_{0}} \Psi_{0}+T_{R}\left(E_{0}\right) T_{\delta V} \delta \Psi^{(1)}\right\rangle=0
$$

Therefore,

$$
\begin{aligned}
\delta E^{(2)}\left\langle T_{V_{0}} \bar{\Psi}_{0}, T_{R}^{\prime}\left(E_{0}\right) T_{V_{0}} \Psi_{0}\right\rangle & =-\left\langle T_{V_{0}} \bar{\Psi}_{0}, T_{R}\left(E_{0}\right) T_{\delta V} \delta \Psi^{(1)}\right\rangle \\
& =-\left\langle\overline{T_{R}\left(E_{0}\right) T_{V_{0}} \Psi_{0}}, T_{\delta V} \delta \Psi^{(1)}\right\rangle \\
& =\left\langle\bar{\Psi}_{0}, T_{\delta V} \delta \Psi^{(1)}\right\rangle .
\end{aligned}
$$

The second equality in (5.13) follows from Lemma 4.3, and the third follows from (4.7). Finally, (5.11) follows from (5.2).

6. The Schrödinger equation with potentials with $N$-fold symmetric (microstructure) potentials. Let $r=|x|$ and $\theta \in[0,2 \pi]$ denote polar coordinates in the plane, $\mathbb{R}^{2}$. We consider $V(x)=V_{0}(r)+\delta V_{N}(x)$, where $V_{0}(r)$ defines the averaged structure and $\delta V_{N}$, the microstructure

$$
\delta V_{N}(x)=\delta \tilde{V}(r, N \theta)
$$

Here, $\delta \tilde{V}(r, \Theta)$ is $2 \pi$-periodic and mean-zero in $\Theta$. Thus we have a structure with $N$-fold rotational symmetry.

We want to show that to any scattering resonance of the averaged structure, there is a nearby scattering resonance of the perturbed structure, provided that $N$ is sufficiently large. In order to apply the results of Theorem 4.1 we must show that, as $N \rightarrow \infty$,

$$
\left\|\delta V_{N}\right\| \mid \equiv T_{\delta V_{N}}\left\|_{\mathcal{B}\left(L^{2}\right)}=\right\|\langle D\rangle^{-1} \delta \check{V}_{N}\langle D\rangle^{-1} \|_{\mathcal{B}\left(L^{2}\right)} \rightarrow 0
$$


where $\delta \check{V}_{N}=\chi^{-1} \delta V_{N} \chi^{-1}$. We next prove this and, in particular, obtain the precise estimate of $\left\|\delta V_{N}\right\| \mid$ in terms of $N$.

TheOREM 6.1. For some positive constant, $C$, depending on $V$ and $\chi$,

$$
\left\|\delta V_{N}\right\| \leq C \frac{1}{N}
$$

Corollary 6.2. By Theorems 6.1 and 4.1, there exists a positive integer, $N_{*}>$ 0 , such that, for $N \geq N_{*}$, the scattering resonance problem for $H=-\Delta+V_{0}+\delta V_{N}$ has a solution $\left(E_{N}, \psi_{N}\right)$ with scattering frequency $E_{N}$ near $E_{0}$.

To prove (6.3), we shall make use of the Fourier series of $\delta V_{N}$ :

$$
\delta V_{N}(\cdot, \theta)=\sum_{k \neq 0} \delta V_{N}{ }^{(k)}(\cdot) e^{i k \theta}
$$

A similar expansion holds for $\delta \check{V}_{N}$, with $\delta V_{N}{ }^{(k)}$ replaced by $\delta \check{V}_{N}{ }^{(k)} \equiv \chi^{-1} \delta V_{N}{ }^{(k)} \chi^{-1}$.

Proposition 6.3. Let $K(\Delta)$ denote a function of the Laplacian. Then,

$$
K(\Delta) e^{i \ell \theta} f(r)=e^{i \ell \theta} K\left(\Delta_{\ell}\right) f(r),
$$

where

$$
\Delta_{\ell}=\Delta_{r}-\frac{\ell^{2}}{r^{2}}
$$

In particular,

$$
\begin{aligned}
\langle D\rangle^{-1} e^{i \ell \theta} f(r) & =e^{i \ell \theta}\left\langle D_{\ell}\right\rangle^{-1} f(r), \\
\left\langle D_{\ell}\right\rangle^{-1} & =\left(m^{2} I-\Delta_{\ell}\right)^{-\frac{1}{2}} .
\end{aligned}
$$

Proof of Theorem 6.1. We now estimate the norm of $T_{\delta V_{N}}$. Let $f \in L^{2}$ be arbitrary. Then,

$$
\begin{aligned}
\langle D\rangle^{-1} \delta \check{V}_{N}\langle D\rangle^{-1} f & =\sum_{\ell}\langle D\rangle^{-1} \delta \check{V}_{N}\langle D\rangle^{-1} f_{\ell} e^{i \ell \theta} \\
& =\sum_{\ell}\langle D\rangle^{-1} \delta \check{V}_{N} e^{i \ell \theta}\left\langle D_{\ell}\right\rangle^{-1} f_{\ell} \\
& =\sum_{\ell, k \neq 0}\langle D\rangle^{-1} \delta \check{V}_{N}{ }^{(k)} e^{i(\ell+k N) \theta}\left\langle D_{\ell}\right\rangle^{-1} f_{\ell} \\
& =\sum_{\ell, k \neq 0} e^{i(\ell+k N) \theta}\left\langle D_{\ell+k N}\right\rangle^{-1} \delta \check{V}_{N}{ }^{(k)}\left\langle D_{\ell}\right\rangle^{-1} f_{\ell} .
\end{aligned}
$$

Taking the $L^{2}$ norm and using orthogonality of $\left\{e^{i \ell \theta}: \ell \in Z\right\}$ we have

$$
\begin{aligned}
& \left\|\langle D\rangle^{-1} \delta \check{V}_{N}\langle D\rangle^{-1} f\right\|_{L^{2}}^{2}=\sum_{\ell, k \neq 0}\left\|\left\langle D_{\ell+k N}\right\rangle^{-1} \delta \check{V}_{N}{ }^{(k)}\left\langle D_{\ell}\right\rangle^{-1} f_{\ell}\right\|_{L^{2}}^{2} \\
& \quad=\sum_{\ell, k \neq 0}\left\|\left\langle D_{\ell+k N}\right\rangle^{-1}\left|\delta \check{V}_{N}{ }^{(k)}\right|^{\frac{1}{2}}\left|\delta \check{V}_{N}{ }^{(k)}\right|^{\frac{1}{2}} \operatorname{sgn}\left(\delta \check{V}_{N}{ }^{(k)}\right)\left\langle D_{\ell}\right\rangle^{-1} f_{\ell}\right\|_{L^{2}}^{2} \\
& \leq \sum_{\ell, k \neq 0}\left\|\left\langle D_{\ell+k N}\right\rangle^{-1}\left|\delta \check{V}_{N}{ }^{(k)}\right|^{\frac{1}{2}}\right\|_{\mathcal{B}\left(L^{2}\right)}^{2}\left\|\left|\delta \check{V}_{N}{ }^{(k)}\right|^{\frac{1}{2}}\left\langle D_{\ell}\right\rangle^{-1}\right\|_{\mathcal{B}\left(L^{2}\right)}^{2}\left\|f_{\ell}\right\|_{L^{2}}^{2} .
\end{aligned}
$$


Since the operators $\left|\delta \check{V}_{N}{ }^{(k)}\right|^{\frac{1}{2}}\left\langle D_{\ell}\right\rangle^{-1}$ and $\left\langle D_{\ell}\right\rangle^{-1}\left|\delta \check{V}_{N}{ }^{(k)}\right|^{\frac{1}{2}}$ are adjoints, their $\mathcal{B}\left(L^{2}\right)$ norms are equal, and we have

$$
\left\|\langle D\rangle^{-1} \delta \check{V}_{N}\langle D\rangle^{-1} f\right\|_{L^{2}}^{2} \leq \sum_{\ell, k \neq 0} \Gamma_{\ell+k N, k} \Gamma_{\ell, k}\left\|f_{\ell}\right\|_{L^{2}}^{2},
$$

where

$$
\Gamma_{q, k}=\left\|\left|\delta \check{V}_{N}{ }^{(k)}\right|^{\frac{1}{2}}\left\langle D_{q}\right\rangle^{-1}\right\|_{\mathcal{B}\left(L^{2}\right)}^{2}
$$

Let

$$
B_{\ell} \equiv\left\langle D_{\ell}\right\rangle=\left(m^{2} I-\Delta_{r}+\frac{\ell^{2}}{r^{2}}\right)^{\frac{1}{2}}
$$

Note that

$$
\Gamma_{q, k}=\left\|\langle x\rangle\left|\delta \check{V}_{N}{ }^{(k)}\right|^{\frac{1}{2}} \cdot\langle x\rangle^{-1} B_{q}^{-1}\right\|_{\mathcal{B}\left(L^{2}\right)}^{2} \leq \gamma_{k}\left\|\langle x\rangle^{-1} B_{q}^{-1}\right\|_{\mathcal{B}\left(L^{2}\right)}^{2}
$$

where

$$
\gamma_{k}=\sup _{x}\left\|\langle x\rangle\left|\delta \check{V}_{N}{ }^{(k)}\right|^{\frac{1}{2}}\right\|_{\infty} \leq \gamma,
$$

where $\gamma$ is independent of $k$ since $V(r, \theta)$ is bounded and has compact support in $x$. Therefore,

$$
\left\|\langle D\rangle^{-1} \delta \check{V}_{N}\langle D\rangle^{-1} f\right\|_{L^{2}}^{2} \leq \gamma^{2} \sum_{\ell, k \neq 0}\left\|\langle x\rangle^{-1} B_{\ell+k N}^{-1}\right\|_{\mathcal{B}\left(L^{2}\right)}^{2}\left\|\langle x\rangle^{-1} B_{\ell}^{-1}\right\|_{\mathcal{B}\left(L^{2}\right)}^{2}\left\|f_{\ell}\right\|_{L^{2}}^{2} .
$$

We shall bound (6.14) using the following proposition.

PROPOSITION 6.4.

$$
\left\|\langle x\rangle^{-1} B_{\ell}^{-1} f\right\|_{L^{2}} \leq\left(1+\ell^{2}\right)^{-\frac{1}{2}}\|f\|_{L^{2}} .
$$

The proof is given in section 8 .

We now conclude our estimation of $\left\|\delta V_{N}\right\|$. By Proposition 6.4 applied to the operators $B_{\ell}$ and $B_{\ell+k N}$ we have from (6.15)

$$
\left\|\langle D\rangle^{-1} \delta V_{N}\langle D\rangle^{-1} f\right\|_{L^{2}}^{2} \leq 2 \gamma^{2} \sum_{|k| \geq 1} \sum_{\ell} \frac{1}{1+(\ell+k N)^{2}} \frac{1}{1+\ell^{2}}\left\|f_{\ell}\right\|_{L^{2}}^{2} .
$$

Consider the above sum over the range $k \geq 1$. The range $k \leq-1$ is treated similarly:

$$
\sum_{k \geq 1} \sum_{\ell} \frac{1}{1+(\ell+k N)^{2}} \frac{1}{1+\ell^{2}}\left\|f_{\ell}\right\|_{L^{2}}^{2}=\sum_{\mathbf{A}}+\sum_{\mathbf{B}}+\sum_{\mathbf{C}} .
$$

Here, we use the notation

$$
\begin{aligned}
& \sum_{\mathbf{A}} \equiv \sum_{k \geq 1} \sum_{-2 k N \leq \ell \leq-k N / 2}\{\cdots\}, \quad \sum_{\mathbf{B}} \equiv \sum_{k \geq 1} \sum_{\ell \geq-k N / 2}\{\cdots\}, \quad \text { and } \\
& \sum_{\mathbf{C}} \equiv \sum_{k \geq 1} \sum_{\ell \leq-2 k N}\{\cdots\} .
\end{aligned}
$$


These three sums are estimated as follows:

$$
\begin{aligned}
\sum_{\mathbf{A}} & \leq \sum_{k \geq 1} \sum_{-2 k N \leq m \leq-k N / 2} \frac{1}{1+\ell^{2}}\left\|f_{\ell}\right\|_{L^{2}}^{2} \leq \sum_{k \geq 1} \frac{1}{1+\frac{k^{2} N^{2}}{4}}\left\|f_{\ell}\right\|_{L^{2}}^{2} \leq C \frac{1}{N^{2}}\|f\|_{L^{2}}^{2}, \\
\sum_{\mathbf{B}} & \leq \sum_{k \geq 1} \frac{1}{1+\frac{k^{2} N^{2}}{4}} \sum_{\ell \geq-k N / 2} \frac{1}{1+\ell^{2}}\left\|f_{\ell}\right\|_{L^{2}}^{2} \leq C \frac{1}{N^{2}}\|f\|_{L^{2}}^{2}, \\
\sum_{\mathbf{C}} & \leq \sum_{k \geq 1} \frac{1}{1+k^{2} N^{2}} \sum_{\ell \leq-2 k N} \frac{1}{1+\ell^{2}}\left\|f_{\ell}\right\|_{L^{2}}^{2} \leq C \frac{1}{N^{2}}\|f\|_{L^{2}}^{2} .
\end{aligned}
$$

This completes the proof of Theorem 6.1.

7. Comparison of perturbation and homogenization expansions. In the previous section, we showed the applicability of resonance perturbation theory to the class of potentials $V_{N}$; see (1.5). In this section, we first summarize the formal homogenization expansion [7], derived and used extensively to obtain an expansion and efficient numerical algorithm for computation of leakage rates (imaginary parts of scattering resonances) for a class of photonic microstructure waveguides. We then prove that this expansion of the scattering resonance frequency, $E\left(V_{N}\right)$, agrees through second order with the expansion

$$
E(V)=E_{0}+\delta E^{(1)}+\delta E^{(2)}
$$

of the previous section.

7.1. Summary of homogenization/multiscale expansion. We begin with a brief summary of the multiscale/homogenization expansion. For more detail, see [7]. We seek solutions of the equation

$$
\left(-\Delta+V_{N}-E\right) \psi=0
$$

that satisfy an outgoing radiation condition at infinity.

For simplicity, we take

$$
V_{N}=V_{N}(r, \theta, N \theta)=V_{0}(r)+\delta \tilde{V}(r, N \theta),
$$

where $\delta \tilde{V}(r, \Theta+2 \pi)=\delta \tilde{V}(r, \Theta)$, and we define

$$
\delta V(r, \theta)=\delta \tilde{V}(r, N \theta) .
$$

The more general case, where $V_{0}$ is not necessarily radial, can also be treated.

As in [7], we view a solution of (7.1) as a function of slow variables $r, \theta$ and a fast variable $\Theta=N \theta$ :

$$
\psi=\Phi(r, \theta, \Theta) .
$$

Equation (7.1) can be rewritten as an equation for $\Phi(r, \theta, \Theta)$ by replacing $\partial_{\theta}^{2}$ in the Laplacian appearing in (7.1) by $\left(\partial_{\theta}+N \partial_{\Theta}\right)^{2}$. We then substitute into the resulting equation the following expansions:

$$
\begin{aligned}
& \Phi^{(N)}=\Phi_{0}+\frac{1}{N} \Phi_{1}+\frac{1}{N^{2}} \Phi_{2}+\frac{1}{N^{3}} \Phi_{3}+\frac{1}{N^{4}} \Phi_{4}+\cdots, \\
& E^{(N)}=E_{0}+\frac{1}{N} E_{1}+\frac{1}{N^{2}} E_{2}^{(\text {homog })}+\cdots
\end{aligned}
$$


Equating like orders of $N^{-1}$ yields a hierarchy of equations of the form

$$
\frac{1}{r^{2}} \partial_{\psi}^{2} \Phi_{j}=\mathcal{F}_{j}, \quad j=0,1,2, \ldots
$$

The solvability condition for (7.6) is

$$
\left\langle\mathcal{F}_{j}\right\rangle_{\mathrm{av}}(r) \equiv \frac{1}{2 \pi} \int_{0}^{2 \pi} \mathcal{F}_{j}(\cdot, \Theta) d \Theta=0 .
$$

This hierarchy can be solved recursively. The equations from $\mathcal{O}\left(N^{2}\right)$ through $\mathcal{O}(1)$ imply that the leading order term is a solution of the scattering resonance problem for $V_{0},\left(E_{0}, \psi_{0}\right)$, which we have taken in (7.5). Furthermore,

$$
\Phi_{2}=\Phi_{2}^{(p)}(r, \theta, \Theta)+\Phi_{2}^{(h)}(r, \theta)
$$

where

$$
\Phi_{2}^{(p)}(r, \theta, \Theta)=\partial_{\Theta}^{-2}\left[\delta \tilde{V} r^{2} \psi_{0}\right] .
$$

At order $\mathcal{O}\left(N^{-1}\right)$, we find $\delta E_{1}=0$ and $\Phi_{1}=0$, and we can solve for $\Phi_{3}$ in terms of $\Phi_{2}^{(p)}$. Finally, $E_{2}^{(\text {homog) }}$ is determined via the solvability condition for the $\Phi_{4}$ equation. This solvability equation reads as

$$
\left(-\Delta-E_{0}+V_{0}\right) \Phi_{2}^{(h)}=E_{2}^{(\mathrm{homog})} \psi_{0}+\left\langle\delta \tilde{V} \partial_{\Theta}^{-2} \delta \tilde{V}\right\rangle_{\mathrm{av}}(r) r^{2} \psi_{0}
$$

Now $E_{2}^{\text {(homog) }}$ is to be chosen so that (7.10) has a solution satisfying an outward going radiation condition at infinity. In [7] we implemented this construction of $E_{2}^{\text {(homog) }}$ and $\Phi_{2}$ for a class of "separable microstructures" as part of a numerical algorithm used to treat general microstructures, $\delta \tilde{V}$.

7.2. $N^{-2}$ corrections: Homogenization vs. resonance perturbation theory. In this subsection we show that the expression for $E_{2}^{(\text {homog) }}$, obtained as a solvability condition for (7.10), is, up to higher order corrections in $N^{-1}$, the same as that given by (5.10) in Lemma 5.1.

We begin by reformulating (7.10) in $L^{2}$. First rewrite (7.10) as

$$
\left(-\Delta-E_{0}+V_{0}\right) \Phi_{2}^{(h)}=-E_{2}^{(\mathrm{homog})} R_{0}(E) V_{0} \psi_{0}+\left\langle\delta \tilde{V} \partial_{\Theta}^{-2} \delta \tilde{V}\right\rangle_{\mathrm{av}}(r) r^{2} \psi_{0},
$$

where we have used the Lippman-Schwinger equation (3.8) to replace $\psi_{0}$ by $-R_{0}\left(E_{0}\right) V_{0} \psi_{0}$.

For $E$ with $\Re E>0$ and $\Im E>0$, consider the equation

$$
\left(-\Delta-E+V_{0}\right) \mathcal{R}=-E_{2}^{(\mathrm{homog})} R_{0}(E) V_{0} \psi_{0}+\left\langle\delta \tilde{V} \partial_{\Theta}^{-2} \delta \tilde{V}\right\rangle_{\mathrm{av}}(r) r^{2} \psi_{0}
$$

$R_{0}(E)$ is a bounded operator on $L^{2}$, and applying it to both sides of (7.12) gives

$$
\begin{aligned}
\left(I+R_{0}(E) V_{0}\right) \mathcal{R}= & -E_{2}^{(\mathrm{homog})} R_{0}(E) R_{0}(E) V_{0} \psi_{0}+R_{0}(E)\left\langle\delta \tilde{V} \partial_{\Theta}^{-2} \delta \tilde{V}\right\rangle_{\mathrm{av}}(r) r^{2} \psi_{0} \\
& -E_{2}^{(\mathrm{homog})} R_{0}^{\prime}(E) V_{0} \psi_{0}+R_{0}(E)\left\langle\delta \tilde{V} \partial_{\Theta}^{-2} \delta \tilde{V}\right\rangle_{\mathrm{av}}(r) r^{2} \psi_{0} \\
\equiv & \mathcal{S} .
\end{aligned}
$$


We then define

$$
\mathcal{R}_{1}=\langle D\rangle \chi \mathcal{R}, \quad \mathcal{S}_{1}=\langle D\rangle \chi \mathcal{S}
$$

and introduce localization and smoothing operators into (7.13) to find

$$
\left(I+T_{R}(E) T_{V_{0}}\right) \mathcal{R}_{1}=\mathcal{S}_{1}
$$

For $E$ in the upper half-plane, (7.14) is always solvable. We now analytically continue across the continuous spectrum, the half-line $E \geq 0$ (corresponding to $\mu \in \mathbb{R}$ ), to the lower half-plane and, in particular, to a small punctured disc about $E=E_{0}$, $0<\left|E-E_{0}\right|<\epsilon$. We note that $\mathcal{S}_{1} \in L^{2}\left(\mathbb{R}^{n}\right)$, since $V_{0}$ and $\left\langle\delta \tilde{V} \partial_{\Theta}^{-2} \delta \tilde{V}\right\rangle_{\text {av }}(r)$ both have compact support. From Corollary 3.6 we have that $T_{R}\left(E_{0}\right) T_{V_{0}}$ is compact, and therefore by Lemma 4.2 the solution $\mathcal{R}_{1}$ of (7.14) exists for $E=E_{0}$ if and only if the following solvability condition holds:

$$
\left\langle T_{V_{0}} \bar{\Psi}_{0}, G\right\rangle=0
$$

We may read off $E_{2}^{\text {(homog) }}$ from (7.14). After recognizing that the coefficient of $E_{2}^{(\text {homog) }}$ is exactly $C_{d E}$ from (5.6), we find that

$$
\begin{aligned}
E_{2}^{\text {(homog) }} & =C_{d E}^{-1}\left\langle T_{V_{0}} \bar{\Psi}_{0}, T_{R}\left(E_{0}\right) T_{\mathcal{V}} \Psi_{0}\right\rangle \\
& =-C_{d E}^{-1}\left\langle\bar{\Psi}_{0}, T_{\mathcal{V}} \Psi_{0}\right\rangle,
\end{aligned}
$$

where we have used Lemma 4.3 and (3.9) and defined

$$
\mathcal{V}(r)=r^{2}\left\langle\delta \tilde{V} \partial_{\Theta}^{-2} \delta \tilde{V}\right\rangle_{\mathrm{av}}(r)
$$

THEOREM 7.1. When $\delta V$ is of the form (7.3) and has compact support,

$$
\left|\delta E^{(2)}-N^{-2} E_{2}^{(\text {homog })}\right|=\mathcal{O}\left(N^{-(2+\tau)}\right),
$$

where $\tau>0$ depends on the details of $\delta V$. In particular, if $\delta V(r, \theta)$ is twice differentiable in $r$, then $\tau=2$; if $\delta V(r, \theta)$ has a finite number of jump discontinuities in $r$, then $\tau=1$.

Proof of Theorem 7.1. For ease of presentation, we make the simplifying, but inessential, assumption that

$$
\psi_{0}=\psi_{0}(r)
$$

We begin by expressing $\delta E^{(2)}$ from (5.11) as

$$
\delta E^{(2)}=-C_{d E}^{-1}\left(N_{2}^{(\mathrm{PT}, \mathrm{main})}-N_{2}^{(\mathrm{PT}, \mathrm{rem})}\right),
$$

where

$$
\begin{aligned}
N_{2}^{(\mathrm{PT}, \text { main })} & =\left\langle T_{\delta V} \bar{\Psi}_{0}, T_{R}\left(E_{0}\right) T_{\delta V} \Psi_{0}\right\rangle, \\
N_{2}^{(\mathrm{PT}, \mathrm{rem})} & =\left\langle T_{\delta V} \bar{\Psi}_{0},\left(I+T_{R}\left(E_{0}\right) T_{V_{0}}\right)^{-1} T_{R}\left(E_{0}\right) T_{V_{0}} T_{R}\left(E_{0}\right) T_{\delta V} \Psi_{0}\right\rangle, \\
C_{d E} & =\left\langle T_{V_{0}} \bar{\Psi}_{0}, T_{R}^{\prime}\left(E_{0}\right) T_{V_{0}} \Psi_{0}\right\rangle .
\end{aligned}
$$


Estimation of the remainder $\boldsymbol{N}_{2}^{(\mathrm{PT}, \mathrm{rem})}$. We begin by removing the smoothing operators:

$$
N_{2}^{(\mathrm{PT}, \mathrm{rem})}=\langle\underbrace{\chi^{-1} V_{0} \overline{R_{0}\left(E_{0}\right)} \delta V \bar{\psi}_{0}}_{f}, \underbrace{\left(I+\chi R_{0}\left(E_{0}\right) V_{0} \chi^{-1}\right)^{-1}}_{A^{-1}} \underbrace{\chi R_{0}\left(E_{0}\right) \delta V \psi_{0}}_{g}\rangle .
$$

By the proof of Proposition 3.1, the operator $\left(I+\chi R_{0}\left(E_{0}\right) V_{0} \chi^{-1}\right)^{-1}$ is meromorphic with finite-rank residues at the poles. Because we are evaluating it at a pole, we must verify that $g \perp \operatorname{ker} A^{*}$ and $f \perp \operatorname{ker} A$. Under our assumption that $\operatorname{ker} A$ is the onedimensional space spanned by $\chi \psi_{0}$, it is easy to see that ker $A^{*}$ is also one-dimensional and spanned by $\chi^{-1} V_{0} \bar{\psi}_{0}$. We may then compute

$$
\begin{aligned}
\left\langle\chi^{-1} V_{0} \bar{\psi}_{0}, g\right\rangle & =\left\langle R_{0}\left(E_{0}\right)^{*} V_{0} \bar{\psi}_{0}, \delta V \psi_{0}\right\rangle \\
& =0,
\end{aligned}
$$

which follows because $R_{0}\left(E_{0}\right)^{*} V_{0} \bar{\psi}_{0}$ is a radial function while $\delta V \psi_{0}$ is mean-zero in $\theta$. Similarly, we may verify that $\left\langle f, \chi \psi_{0}\right\rangle=0$ and conclude that

$$
\begin{aligned}
\left\langle f, A^{-1} g\right\rangle & \leq C\|f\|_{2}\|g\|_{2} \\
& =C\left\|\chi \chi^{-1} V_{0} \chi^{-1} \chi R_{0}\left(E_{0}\right)^{*} \delta V \bar{\psi}_{0}\right\|_{2}\left\|\chi R_{0}\left(E_{0}\right) \delta V \psi_{0}\right\|_{2} \\
& \leq C\left\|\chi R_{0}\left(E_{0}\right) \delta V \psi_{0}\right\|_{2}^{2} .
\end{aligned}
$$

In order to bound $\left\|\chi R_{0}\left(E_{0}\right) \delta V \psi_{0}\right\|_{2}^{2}$, we first express $R_{0}\left(E_{0}\right)$ as a sum of a bounded operator and an unbounded correction, using the analytic continuation formula (3.20) with $j=0$. This gives

$$
\left\|\chi R_{0}\left(\mu_{0}\right) \delta V \psi_{0}\right\|_{2} \leq\left\|\chi R_{0}\left(-\mu_{0}\right) \delta V \psi_{0}\right\|_{2}+C\left\|\chi J_{0}\left(\mu_{0}|\cdot|\right) \star \delta V \psi_{0}\right\|_{2} .
$$

We next expand $\delta V$ in a Fourier series

$$
\delta V(r, \theta)=\sum_{n \neq 0} \delta V_{n}(r) e^{i n N \theta} .
$$

We consider the first term in (7.19) and use Proposition 6.3 to see that

$$
\chi R_{0}\left(-\mu_{0}\right) \delta V_{n} \psi_{0} e^{i n N \theta}=e^{i n N \theta} \chi G_{n N}\left(\mu_{0}\right) \delta V_{n} \psi_{0},
$$

where

$$
G_{\ell}(\mu)=\left(-\Delta_{r}+\frac{(n N)^{2}}{r^{2}}-\mu_{0}^{2}\right)^{-1}
$$

is the partial wave Green's function. In section 8, we prove the following estimate on $G_{\ell}(\mu)$.

Proposition 7.2. Let $\mu \in \mathbb{C} \backslash\{0\}$ with $|\Im \mu|<M$. There exists a constant, $C$, such that for all $l \geq 1$

$$
f \in L^{2}\left(\mathbb{R}^{2}\right) \Longrightarrow\left\|\chi G_{\ell}(\mu) \chi f\right\|_{2} \leq C \frac{\|f\|_{2}}{\ell^{2}} .
$$

This result implies that

$$
\left\|\chi R_{0}\left(-\mu_{0}\right) \delta V_{n} \psi_{0} e^{i n N \theta}\right\|_{2} \leq C \frac{\left\|\chi^{-1} \delta V_{n} \psi_{0}\right\|_{2}}{(n N)^{2}},
$$


and therefore

$$
\begin{aligned}
\left\|\chi R_{0}\left(-\mu_{0}\right) \delta V \psi_{0} e^{i n N \theta}\right\|_{2} & \leq C N^{-2} \sup _{n}\left\|\chi^{-1} \delta V_{n} \psi_{0}\right\|_{2} \\
& \leq C N^{-2} .
\end{aligned}
$$

We still must bound the second term in (7.19). We observe that for $f \in L^{2}\left(\mathbb{R}^{2}\right)$ and compact support,

$$
\begin{aligned}
J_{0}\left(\mu_{0}|\cdot|\right) \star f(x) & =\int d y J_{0}\left(\mu_{0}|x-y|\right) f(y) \\
& =2 \pi \int d y \int_{S^{1}} d \omega e^{i \mu_{0}(x-y) \omega} f(y) \\
& =2 \pi \int_{S^{1}} d \omega e^{i \mu_{0} x \omega} \hat{f}\left(\mu_{0} \omega\right),
\end{aligned}
$$

and therefore

$$
\begin{aligned}
\left\|\chi J_{0}\left(\mu_{0}|\cdot|\right) \star f\right\|_{2} & \leq C \sup _{\omega \in S^{1}}\left|\hat{f}\left(\mu_{0} \omega\right)\right|\left\|\chi(x) \sup _{\omega \in S^{1}}\left|e^{i \mu_{0} x \omega}\right|\right\|_{2} \\
& \leq C \sup _{\omega \in S^{1}}\left|\hat{f}\left(\mu_{0} \omega\right)\right| .
\end{aligned}
$$

Next, we will need the following lemma.

LEMMA 7.3. If $F(r, \theta)=f(r) e^{i l \theta}$, then its Fourier transform $\hat{F}$ can be written in polar coordinates $(\rho, \nu)$ as

$$
\hat{F}(\rho, \nu)=2 \pi e^{i l(\nu+3 \pi / 2)} \int_{0}^{\infty} r d r f(r) J_{l}(\rho r) .
$$

The proof is given in Appendix B.

Because we are considering the limit of large $N$ while $\mu_{0}$ is fixed, we may use the asymptotic form (A.6) of $J_{n}$ along with (7.22) and Lemma 7.3 to find that

$$
\begin{aligned}
\left\|\chi J_{0}\left(\mu_{0}|\cdot|\right) \star \delta V \psi_{0}\right\|_{2} & \leq \sum_{n}\left\|\chi J_{0}\left(\mu_{0}|\cdot|\right) \star \delta V_{n} e^{i n N \theta} \psi_{0}\right\|_{2} \\
& \leq C \sum_{n} \int r d r\left|\delta V_{n}(r) \psi_{0}(r) J_{n N}\left(\left|\mu_{0}\right| r\right)\right| \\
& \leq \sum_{n} \frac{\left(C\left|\mu_{0}\right|\right)^{n N}}{(n N) !} \\
& \leq \frac{\left(C\left|\mu_{0}\right|\right)^{N}}{N !}
\end{aligned}
$$

$N_{2}^{(\mathrm{PT}, \text { main })}=2$ nd order homogenization + small correction. In the expression for $N_{2}^{(\mathrm{PT}, \text { main })},(7.17)$, we first remove the smoothing operators and move to the physical sheet using the analytic continuation formula, (3.20), shedding a small correction in the process:

$$
\begin{aligned}
N_{2}^{(\mathrm{PT}, \text { main })} & =\left\langle\delta V \bar{\psi}_{0}, R_{0}\left(\mu_{0}\right) \delta V \psi_{0}\right\rangle \\
& =\left\langle\delta V \bar{\psi}_{0}, R_{0}\left(-\mu_{0}\right) \delta V \psi_{0}\right\rangle+\frac{i}{2}\left\langle\delta V \bar{\psi}_{0}, J_{0}\left(\mu_{0}|\cdot|\right) \delta V \psi_{0}\right\rangle
\end{aligned}
$$


In order to show that the second term of (7.24) is small, we note that for $f, g \in L^{2}\left(\mathbb{R}^{2}\right)$

$$
\begin{aligned}
\left\langle f, J_{0}(\mu|\cdot|) g\right\rangle & =\int d x d y \bar{f}(x) J_{0}(\mu|x-y|) g(y) \\
& =\int_{S^{1}} d \omega \int d x e^{i \mu x \cdot \omega} \bar{f}(x) \int d y e^{-i \mu y \cdot \omega} g(y) \\
& =\int_{S^{1}} d \omega \hat{f}(\mu \omega) \hat{g}(\mu \omega),
\end{aligned}
$$

and therefore, using (7.23),

$$
\left|\left\langle\delta V \bar{\psi}_{0}, J_{0}\left(\mu_{0}|\cdot|\right) \delta V \psi_{0}\right\rangle\right| \leq \frac{(C|\mu|)^{2 N}}{(N !)^{2}}
$$

In order to estimate the difference between the first term in (7.24) and $\left\langle\bar{\Psi}_{0}, T_{\mathcal{V}} \Psi_{0}\right\rangle$, the numerator from (7.16), we first prove the following lemma.

LEMMA 7.4.

$$
\begin{aligned}
\left\langle\delta V \bar{\psi}_{0}, R_{0}\left(-\mu_{0}\right) \delta V \psi_{0}\right\rangle & =\sum_{n \neq 0}\left\langle f_{n}^{(L)}, R_{0}\left(-\mu_{0}\right) f_{n}^{(R)}\right\rangle, \\
N^{-2}\left\langle\bar{\Psi}_{0}, T_{\mathcal{V}} \Psi_{0}\right\rangle & =\sum_{n \neq 0}\left\langle f_{n}^{(L)}, r^{2} \partial_{\theta}^{-2} f_{n}^{(R)}\right\rangle
\end{aligned}
$$

where

$$
\begin{aligned}
& f_{n}^{(L)}(r, \theta)=\delta V_{n}(r) \bar{\psi}_{0}(r) e^{i n N \theta} \\
& f_{n}^{(R)}(r, \theta)=\delta V_{n}(r) \psi_{0}(r) e^{i n N \theta} .
\end{aligned}
$$

Proof of Lemma 7.4. We first note that (7.25) follows immediately on expanding $\delta V$ in a Fourier series (7.20) and moving to a Fourier integral representation, using (7.23).

In order to see (7.26), we begin by writing

$$
N^{-2}\left\langle\bar{\Psi}_{0}, T_{\mathcal{V}} \Psi_{0}\right\rangle=\left\langle\delta V \bar{\psi}_{0}, r^{2} \partial_{\theta}^{-2} \delta V \psi_{0}\right\rangle,
$$

which follows directly from the definitions. We may then expand $\delta V(r, \theta)$ in a Fourier series (7.20) and observe that, again, the off-diagonal terms vanish.

We may now complete the proof of Theorem 7.1. We begin by using Lemma 7.4 to see that

$$
\begin{aligned}
\mid \delta E^{(2)}-N^{-2} E_{2}^{(\text {homog) }) \mid=} & C_{d E}^{-1} \mid\left\langle\delta V \bar{\psi}_{0}, R_{0}\left(-\mu_{0}\right) \delta V \psi_{0}\right\rangle \\
& -N^{-2}\left\langle\bar{\Psi}_{0}, T_{\mathcal{V}} \Psi_{0}\right\rangle \mid+\mathcal{O}\left(N^{-4}\right) \\
\leq & C_{d E}^{-1} \sum_{n \neq 0}\left|\left\langle f_{n}^{(L)},\left(R_{0}\left(-\mu_{0}\right)-r^{2} \partial_{\theta}^{-2}\right) f_{n}^{(R)}\right\rangle\right|+\mathcal{O}\left(N^{-4}\right) .
\end{aligned}
$$

We may rewrite the summand in (7.27) as

$$
\begin{aligned}
& \left|\left\langle f_{n}^{(L)},\left(R_{0}\left(-\mu_{0}\right)-r^{2} \partial_{\theta}^{-2}\right) f_{n}^{(R)}\right\rangle\right| \\
& \quad=\left|\left\langle f_{n}^{(L)},\left(-\Delta_{r}+\frac{(n N)^{2}}{r^{2}}-\mu_{0}^{2}\right)^{-1}\left(\Delta_{r}+\mu_{0}^{2}\right)\left(\frac{r^{2}}{(n N)^{2}}\right) f_{n}^{(R)}\right\rangle\right| .
\end{aligned}
$$


In order to estimate (7.28), we prove in section 8 the following proposition.

Proposition 7.5. When $f, g \in L_{\infty}([0, R]), g \in C^{2}$, and $\ell$ is taken large depending on $\mu$ and $R$,

$$
\left|\left\langle f, G_{\ell}(\mu) \Delta_{r} g\right\rangle\right| \leq \frac{C}{\ell^{2}}\|f\|_{\infty}\left\|\Delta_{r} g\right\|_{\infty},
$$

while when $g$ is piecewise $C^{2}$, with a finite number of jump discontinuities in $g$ or $g^{\prime}$, then

$$
\left|\left\langle f, G_{\ell}(\mu) \Delta_{r} g\right\rangle\right| \leq \frac{C}{\ell}\|f\|_{\infty}\|g\|_{\infty}
$$

By Proposition 7.5

$$
|(7.28)| \leq C \frac{1}{(n N)^{2}} \frac{1}{(n N)^{\tau}}
$$

Use of this bound in (7.27) and summing over $n \neq 0$ implies that

$$
\left|\delta E^{(2)}-N^{-2} E_{2}^{(\text {homog })}\right|=\mathcal{O}\left(N^{-2-\tau}\right)+\mathcal{O}\left(N^{-4}\right) .
$$

This completes the proof of Theorem 7.1.

8. Partial wave Green's function estimates. In this section we prove the weighted estimates, used in sections 6 and 7, involving the partial wave Green's function

$$
G_{\ell}(\mu)=\left(-\Delta_{r}+\frac{\ell^{2}}{r^{2}}-\mu^{2}\right)^{-1},
$$

defined for $\mu$ in the physical upper half-plane. $G_{\ell}(\mu)$ may be analytically continued to the lower half-plane by explicitly computing its kernel [16]:

$$
\begin{aligned}
G_{\ell}(\mu) f(r) & =\int_{0}^{\infty} G_{\ell}\left(r, r^{\prime} ; \mu\right) f\left(r^{\prime}\right) r^{\prime} d r^{\prime}, \\
G_{\ell}\left(r, r^{\prime} ; \mu\right) & =-\frac{\pi}{2} i\left\{\begin{array}{l}
J_{\ell}(\mu r) H_{\ell}^{(1)}\left(\mu r^{\prime}\right) \text { if } r^{\prime} \geq r, \\
H_{\ell}^{(1)}(\mu r) J_{\ell}\left(\mu r^{\prime}\right) \text { if } r \geq r^{\prime} .
\end{array}\right.
\end{aligned}
$$

We first prove Propositions 7.2 and 7.5, which are used in section 7. We then prove Proposition 6.4.

Proof of Proposition 7.2. Because the kernel $A\left(r, r^{\prime}\right)=\chi(r) G_{\ell}\left(r, r^{\prime}\right) \chi\left(r^{\prime}\right)$ is symmetric, we may estimate the $L^{2} \rightarrow L^{2}$ norm of the associated operator [4] as

$$
\|A\| \leq \sup _{r} \int r^{\prime} d r^{\prime}\left|A\left(r, r^{\prime}\right)\right|
$$

and use the explicit form (8.1) of $G_{\ell}$. We define

$$
\begin{aligned}
& A_{J}\left(r_{1}, r_{2}\right)=\chi\left(r_{2}\right)\left|H_{\ell}^{(1)}\left(r_{2}\right)\right| \int_{r_{1}}^{r_{2}} r d r\left|J_{\ell}(\mu r)\right| \chi(r), \\
& A_{H}\left(r_{1}, r_{2}\right)=\chi\left(r_{1}\right)\left|J_{\ell}\left(r_{1}\right)\right| \int_{r_{1}}^{r_{2}} r d r\left|H_{\ell}^{(1)}(\mu r)\right| \chi(r),
\end{aligned}
$$

and so

$$
\|A\| \leq \sup _{r^{\prime}}\left(A_{J}\left(0, r^{\prime}\right)+A_{H}\left(r^{\prime}, \infty\right)\right)
$$


It suffices to obtain the bound $\sup _{r_{1}, r_{2}} A_{J, H}\left(r_{1}, r_{2}\right) \leq C \ell^{-2}$. We treat $A_{J}$; the bounds on $A_{H}$ are similar. We prove this bound by separately considering the cases: for $r_{1} \leq r_{2} \leq \sigma, r_{1} \leq \sigma \leq r_{2}$ and $\sigma \leq r_{1} \leq r_{2}$, where $\sigma>0$. We shall see that the dominant contribution to the bound comes from $r$ small, which dictates that $\sigma$ be sufficiently small, yet large enough as $\ell \rightarrow \infty$ so that the large $\ell$ asymptotics in the crossover and large $r$ regions can be used. We set $\sigma=\sqrt{\ell}$.

$\sqrt{\ell} \leq r_{1} \leq r_{2}$ : An examination of the asymptotic forms (A.1) reveals that the following bounds hold for $r$ bounded away from the origin. For $r \geq r_{\#}$, there exists $C_{\#}>0$, such that

$$
\begin{aligned}
\chi(r)\left|J_{\ell}(\mu r)\right| & \leq C_{\#} e^{-(M-|\Im \mu|) r}, \\
\chi(r)\left|H_{\ell}(\mu r)\right| & \leq C_{\#} e^{-(M-\Im \mu) r} .
\end{aligned}
$$

Therefore,

$$
\begin{aligned}
A_{J}\left(r_{1}, r_{2}\right) & \leq C e^{-(M-\Im \mu) r_{2}} \int_{r_{1}}^{r_{2}} r d r e^{-(M-|\Im \mu|) r} \\
& \leq C e^{-(1-\epsilon)(M-\Im \mu) r_{2}} .
\end{aligned}
$$

Therefore, if $r_{2}>r_{1}>\sqrt{\ell}$, then

$$
A_{J, H}\left(r_{1}, r_{2}\right) \leq C e^{-C^{\prime} \sqrt{l}} \leq C \ell^{-2} .
$$

$r_{1} \leq r_{2} \leq \sqrt{\ell}$ : Note that the asymptotic expressions (A.6) hold, and therefore

$$
\begin{aligned}
A_{J}\left(r_{1}, r_{2}\right) & \leq C(\ell-1) ! r_{2}^{-1} e^{-M r_{2}} \int_{r_{1}}^{r_{2}} r d r \frac{r^{\ell}}{\ell !} e^{-M r} \\
& \leq \frac{C}{\ell} r_{2}^{-\ell} e^{-M r_{2}} \int_{r_{1}}^{r_{2}} d r r^{\ell+1} \\
& \leq \frac{C}{\ell^{2}} r_{2}^{2} e^{-M r_{2}} \leq \frac{C}{\ell^{2}} .
\end{aligned}
$$

Finally, we treat the transition region.

$r_{1} \leq \sqrt{\ell} \leq r_{2}$ :

$$
A_{J}\left(r_{1}, r_{2}\right) \leq e^{-(M-|\Im \mu|) r_{2}} \int_{0}^{\sqrt{\ell}} r d r \frac{r^{\ell}}{\ell !}=o\left(e^{-C \sqrt{\ell}}\right) \leq \mathcal{O}\left(\ell^{-2}\right)
$$

This completes the proof of Proposition 7.2.

Proof of Proposition 7.5. Because $f(r)$ and $g(r)$ are supported for $r \in[0, R]$, we may write

$$
\begin{aligned}
\left\langle f, G_{\ell}(\mu) \Delta_{r} g\right\rangle= & \int_{0}^{R} r d r \bar{f}(r) \int_{0}^{R} \tilde{r} d \tilde{r} G(r, \tilde{r})\left(\Delta_{r} g\right)(\tilde{r}) \\
= & C \int_{0}^{R} r d r \bar{f}(r) H_{\ell}^{(1)}(\mu r) \int_{0}^{r} \tilde{r} d \tilde{r} J_{\ell}(\mu r)\left(\Delta_{r} g\right)(\tilde{r}) \\
& +C \int_{0}^{R} r d r \bar{f}(r) J_{\ell}(\mu r) \int_{r}^{R} \tilde{r} d \tilde{r} H_{\ell}^{(1)}(\mu r)\left(\Delta_{r} g\right)(\tilde{r}) \\
\equiv & D_{1}+D_{2} .
\end{aligned}
$$


Case 1. $g \in C^{2}$ : We may use (8.1) and (A.6) to find that

$$
\begin{aligned}
&\left|D_{1}\right|+\left|D_{2}\right| \leq \frac{C}{\ell}[ \int_{0}^{R} r d r|f(r)| \frac{(\ell-1) !}{(\mu r)^{\ell}} \int_{0}^{r} \tilde{r} d \tilde{r} \frac{(\mu \tilde{r})^{\ell}}{\ell !}\left|\Delta_{r} g(\tilde{r})\right| \\
&\left.+\int_{0}^{R} r d r|f(r)| \frac{(\mu r)^{\ell}}{\ell !} \int_{r}^{R} \tilde{r} d \tilde{r} \frac{(\ell-1) !}{(\mu \tilde{r})^{\ell}}\left|\Delta_{r} g(\tilde{r})\right|\right] \\
& \leq \frac{C}{\ell^{2}}\|f\|_{\infty}\left\|\Delta_{r} g\right\|_{\infty},
\end{aligned}
$$

which is the desired bound (7.29).

Case 2. $g$ or $g^{\prime}$ have jump discontinuities: When $g$ or $g^{\prime}$ has a finite number of jump discontinuities at the points $r_{i}$, we introduce a partition of unity $1=\sum_{i} \chi_{i}(r)$, where each $\chi_{i} \in C^{\infty}$ and supp $\chi_{i}$ contains $r_{i}$ and no other breakpoint $r_{j \neq i}$. We may then represent $g$ as

$$
g=\sum_{i} \chi_{i}\left(g_{i}+\theta\left(r-r_{i}\right) \tilde{g}_{i}\right)
$$

where $g_{i}, \tilde{g}_{i} \in C^{2}$ and $\theta(x)$ is the Heaviside function $(\theta(x)=0, x \leq 0$, and $\theta(x)=$ $1, x \geq 0)$. We will examine $D_{1}$ in detail; $D_{2}$ will give a similar contribution, as can be easily checked. Using (8.1),

$$
D_{1}=C \sum_{i} \int_{0}^{R} r d r \bar{f}(r) H_{\ell}^{(1)}(r) \int_{0}^{r} \tilde{r} d \tilde{r} J_{\ell}(\tilde{r}) \Delta_{\tilde{r}}\left(\chi_{i}(\tilde{r})\left(g_{i}(\tilde{r})+\theta\left(\tilde{r}-r_{i}\right) \tilde{g}(\tilde{r})\right)\right) .
$$

A number of terms result from the action of the $\Delta_{\tilde{r}}$ in the inner integral. When all of the $\tilde{g}_{i}\left(r_{i}\right)=0$, i.e., the case of jumps in $g^{\prime}$ only, the result is the same as the $C^{2}$ case (7.29). When some of the $\tilde{g}_{i}\left(r_{i}\right) \neq 0$, however, the sum (8.5) can be written as a dominant term containing $\theta^{\prime \prime}=\delta^{\prime}$, where $\delta(r)$ denotes the one-dimensional Dirac delta distribution, plus contributions satisfying the same bound as in the $C^{2}$ case. That is,

$$
\begin{aligned}
D_{1}= & C \sum_{i} \int_{0}^{R} r d r \bar{f}(r) H_{\ell}^{(1)}(\mu r) \int_{0}^{r} \tilde{r} d \tilde{r} J_{\ell}(\mu \tilde{r}) \chi_{i}(\tilde{r}) \tilde{g}_{i}(\tilde{r}) \delta^{\prime}\left(\tilde{r}-r_{i}\right) \\
& +\mathcal{O}\left(\ell^{-2}\right)\|f\|_{\infty}\|g\|_{\infty} .
\end{aligned}
$$

The sum in (8.6) is, in turn, dominated by the contribution from $\partial_{r} J_{\ell}$ :

$$
\begin{aligned}
\left|D_{1}\right| & \leq C\|f\|_{\infty} \sum_{i}\left\|\tilde{g}_{i}\right\|_{\infty}\left[\int_{0}^{R} r d r\left|H_{\ell}^{(1)}(\mu r)\right| \theta\left(r-r_{i}\right) r_{i} \tilde{g}_{i}\left(r_{i}\right)\left|\partial_{r} J_{\ell}(\mu r)\right|_{r=r_{i}}+\mathcal{O}\left(\ell^{-2}\right)\right] \\
& \leq C\|f\|_{\infty} \sum_{i}\left\|\tilde{g}_{i}\right\|_{\infty}\left[\left(\frac{\ell r_{i}^{\ell-1}}{\ell !}\right) \int_{r_{i}}^{R} r d r \frac{(\ell-1) !}{r^{\ell}}+\mathcal{O}\left(\ell^{-2}\right)\right] \\
& \leq C\|f\|_{\infty} \sum_{i}\left\|\tilde{g}_{i}\right\|_{\infty}\left[\left(\frac{r_{i}}{\ell}\right)+\mathcal{O}\left(\ell^{-2}\right)\right] \\
& \leq \frac{C}{\ell}\|f\|_{\infty}\|g\|_{\infty} .
\end{aligned}
$$

$D_{2}$ may be estimated by similar arguments.

We now turn to the proof of Proposition 6.4, used in section 6. We first need the following proposition. 
Proposition 8.1. Let $V_{0}$ be a nonnegative, bounded, and sufficiently decaying function. For any $p \in \mathbb{C}$, with $\Re p>0$, and $f \in L^{2}\left(\mathbb{R}^{2}\right)$,

$$
\left\|\langle x\rangle^{-1}\left(-\Delta_{r}+\frac{m^{2}}{r^{2}}+V_{0}+p\right)^{-1} f\right\|_{L^{2}} \leq \frac{1}{\Re p+m^{2}}\|f\|_{L^{2}} .
$$

Remark 8.2. We use this result for the case $V_{0}=0$ only. From the proof, it is clear that it holds under much less stringent hypotheses on $V_{0}$.

Proof of Proposition 8.1. The proof is broken into two parts. We first prove estimates for the heat kernel $\exp \left[\left(\Delta-\frac{m^{2}}{r^{2}}\right) t\right]$ and then reduce the desired resolvent estimate to the heat kernel estimate.

Part 1. Let $u_{m}$ denote the solution of the initial value problem for the heat equation:

$$
\partial_{t} u_{m}=\left(\Delta_{r}-\frac{m^{2}}{r^{2}}-V_{0}\right) u_{m}, \quad u_{m}(0)=f .
$$

Multiplication by $u_{m}$ and integration over $\mathbb{R}^{2}$ gives the identity:

$$
\frac{d}{d t} \int\left|u_{m}\right|^{2} d x=-2 \int\left|\nabla u_{m}\right|^{2} d x-2 m^{2} \int \frac{\left|u_{m}\right|^{2}}{r^{2}} d x-2 \int V_{0}\left|u_{m}\right|^{2} d x .
$$

Since $V_{0}$ is nonnegative we can drop both the first and third terms on the right-hand side of (8.9) to obtain

$$
\frac{d}{d t} \int\left|u_{m}\right|^{2} d x \leq-2 m^{2} \int \frac{\left|u_{m}\right|^{2}}{r^{2}} d x .
$$

Integration of (8.10) over the time interval from 0 to $t$ and using that $\left(1+r^{2}\right)^{-1} \leq 1$ yields

$$
\begin{aligned}
& \alpha(t) \leq\|f\|_{L^{2}}^{2}-2 m^{2} \int_{0}^{t} \alpha(s) d s, \\
& \alpha(t) \equiv \int \frac{\left|u_{m}\right|^{2}}{1+r^{2}} d x .
\end{aligned}
$$

It follows that

$$
\left\|\langle x\rangle^{-1} e^{\left(\Delta_{r}-\frac{m^{2}}{r^{2}}-V_{0}\right) t} f\right\|_{L^{2}} \leq e^{-m^{2} t}\|f\|_{L^{2}} .
$$

Part 2. First note that the resolvent and heat kernels are related by the Laplace transform: when $\Re p>0$,

$$
\left(\Delta_{r}-\frac{m^{2}}{r^{2}}-V_{0}-p\right)^{-1} f=\int_{0}^{\infty} e^{-p t} e^{\left(\Delta_{r}-\frac{m^{2}}{r^{2}}-V_{0}\right) t} d t f
$$

Thus, using the weighted estimate (8.12) we have

$$
\begin{aligned}
\|\langle x\rangle^{-1} & \left(\Delta_{r}-\frac{m^{2}}{r^{2}}-V_{0}-p\right)^{-1} f \|_{L^{2}} \\
& \leq \int_{0}^{\infty} e^{-(\Re p) t}\left\|\langle x\rangle^{-1} e^{\left(\Delta_{r}-\frac{m^{2}}{r^{2}}-V_{0}\right) t} f\right\|_{L^{2}} d t \\
& \leq \int_{0}^{\infty} e^{-(\Re p) t} e^{-m^{2} t}\|f\|_{L^{2}}=\frac{1}{\Re p+m^{2}}\|f\|_{L^{2}} .
\end{aligned}
$$


This completes the proof of Proposition 8.1.

Proof of Proposition 6.4. Recall that

$$
B_{\ell}^{2}=m^{2} I-\Delta_{r}+\frac{\ell^{2}}{r^{2}} .
$$

We take $V_{0}=0$ and $p=1$. In this case, there is no restriction on $\ell$ because $\Re p+\ell^{2}=$ $1+\ell^{2}$ is always positive. This gives the bound

$$
\left\|\langle x\rangle^{-1} B_{\ell}^{-2} f\right\|_{L^{2}} \leq \frac{1}{1+\ell^{2}}\|f\|_{L^{2}}
$$

We now deduce the estimates for $B_{\ell}^{-1}$ from those obtained for $B_{\ell}^{-2}$ through the square root formula

$$
B^{-1}=\frac{1}{\pi} \int_{0}^{\infty} \frac{1}{\sqrt{w}} \frac{1}{B^{2}+w} d w
$$

From (8.16) it follows that

$$
\left\|\langle x\rangle^{-1} B_{\ell}^{-1}\right\|_{\mathcal{B}\left(L^{2}\right)} \leq \frac{1}{\pi} \int_{0}^{\infty} \frac{1}{\sqrt{w}}\left\|\frac{1}{\langle x\rangle} \frac{1}{B_{\ell}^{2}+w}\right\|_{\mathcal{B}\left(L^{2}\right)} d w .
$$

By Proposition 8.1, with $V_{0}=0$ and $p=w \geq 0$, we have

$$
\left\|\frac{1}{\langle x\rangle} \frac{1}{B_{\ell}^{2}+w}\right\|_{\mathcal{B}\left(L^{2}\right)} \leq \frac{1}{\ell^{2}+w}
$$

Therefore,

$$
\left\|\langle x\rangle^{-1} B_{\ell}^{-1}\right\|_{\mathcal{B}\left(L^{2}\right)} \leq \frac{1}{\pi} \int_{0}^{\infty} \frac{1}{\sqrt{w}} \frac{1}{\ell^{2}+w} d w \leq \frac{C}{\left(1+\ell^{2}\right)^{1 / 2}}
$$

Appendix A. Asymptotics of Bessel and Hankel functions. We briefly record the well-known asymptotics of Bessel and Hankel functions.

(1) When $\ell \rightarrow \infty$, the following asymptotic expansions hold uniformly for $|\arg z|<$ $\pi-\epsilon$, with $\epsilon>0$ :

$$
\begin{gathered}
J_{\ell}(\ell z) \sim\left(\frac{4 \zeta}{1-z^{2}}\right)^{\frac{1}{4}}\left(\frac{\operatorname{Ai}\left(\ell^{2 / 3} \zeta\right)}{\ell^{1 / 3}} \sum_{k=0}^{\infty} \frac{a_{k}(\zeta)}{\ell^{2 k}}+\frac{\operatorname{Ai}^{\prime}\left(\ell^{2 / 3} \zeta\right)}{\ell^{5 / 3}} \sum_{k=0}^{\infty} \frac{b_{k}(\zeta)}{\ell^{2 k}}\right), \\
H_{\ell}^{(1)}(\ell z) \sim 2 e^{\frac{-\pi i}{3}}\left(\frac{4 \zeta}{1-z^{2}}\right)^{\frac{1}{4}}\left(\frac{\operatorname{Ai}\left(e^{2 \pi i / 3} \ell^{2 / 3} \zeta\right)}{\ell^{1 / 3}} \sum_{k=0}^{\infty} \frac{a_{k}(\zeta)}{\ell^{2 k}}\right. \\
\left.+\frac{e^{2 \pi i / 3} \operatorname{Ai}^{\prime}\left(e^{2 \pi i / 3} \ell^{2 / 3} \zeta\right)}{\ell^{5 / 3}} \sum_{k=0}^{\infty} \frac{b_{k}(\zeta)}{\ell^{2 k}}\right),
\end{gathered}
$$

where

$$
\frac{2}{3} \zeta^{\frac{3}{2}}=\ln \frac{1+\sqrt{1-z^{2}}}{z}-\sqrt{1-z^{2}}
$$

and $a_{k}, b_{k}$ are defined in [1]. 
(2) In the limit $|z| \rightarrow \infty$ with $\ell$ held fixed, these reduce to

$$
\begin{aligned}
J_{\nu}(z) & =\left(\frac{2}{\pi z}\right)^{\frac{1}{2}} \cos \left(z-\frac{1}{2} \nu \pi-\frac{\pi}{4}\right)+e^{|\Im z|} \mathcal{O}\left(|z|^{-1}\right), \quad|\arg z|<\pi \\
Y_{\nu}(z) & =\left(\frac{2}{\pi z}\right)^{\frac{1}{2}} \sin \left(z-\frac{1}{2} \nu \pi-\frac{\pi}{4}\right)+e^{|\Im z|} \mathcal{O}\left(|z|^{-1}\right), \quad|\arg z|<\pi \\
H_{\nu}^{(1)}(z) & =J_{\nu}(z)+i Y_{\nu}(z) \\
& \sim\left(\frac{2}{\pi z}\right)^{\frac{1}{2}} \exp \left(i\left(z-\frac{1}{2} \nu \pi-\frac{\pi}{4}\right), \quad-\pi<\arg z<2 \pi\right. \\
H_{\nu}^{(2)}(z) & =J_{\nu}(z)-i Y_{\nu}(z) \\
& \sim\left(\frac{2}{\pi z}\right)^{\frac{1}{2}} \exp \left(-i\left(z-\frac{1}{2} \nu \pi-\frac{\pi}{4}\right), \quad-2 \pi<\arg z<\pi\right.
\end{aligned}
$$

(3) For $|z| \leq C \sqrt{\ell}$ and $\ell \rightarrow \infty$, (A.1) and the asymptotics or Airy functions [1] imply that

$$
J_{\ell}(z) \sim \frac{1}{\ell !}\left(\frac{z}{2}\right)^{\ell}, \quad H_{\ell}^{(1)}(a) \sim-\frac{i}{\pi}(\ell-1) !\left(\frac{z}{2}\right)^{-\ell} .
$$

Appendix B. Proof of Lemma 7.3. To prove (7.23) we represent $x, k \in \mathbb{R}^{2}$ in polar coordinates as, respectively, $(r, \theta)$ and $(\rho, \nu)$. We wish to compute the Fourier transform

$$
\hat{F}(k)=\int d x e^{-i k \cdot x} F(x), \quad \text { where } F(x)=f(r) e^{i \ell \theta} .
$$

We define $\tilde{\theta}=\theta-\nu$, the angle between $x$ and $k$, and compute as follows:

$$
\begin{aligned}
\hat{F}(k) & =\int_{0}^{\infty} r d r \int_{0}^{2 \pi} d \tilde{\theta} e^{-i \rho r \cos \tilde{\theta}} f(r) e^{i \ell(\tilde{\theta}+\nu)} \\
& =e^{i \ell(\nu+\pi)} \int_{0}^{\infty} f(r) r d r \int_{-\pi}^{\pi} d \tilde{\theta} e^{i \rho r \cos \psi+i \ell \psi} \\
& =2 \pi e^{i \ell(\nu+3 \pi / 2)} \int_{0}^{\infty} f(r) J_{\ell}(\rho r) r d r
\end{aligned}
$$

where we have used (1.17).

Acknowledgments. The authors acknowledge stimulating discussions with R. V. Kohn, G. C. Papanicolaou, and M. Vogelius.

\section{REFERENCES}

[1] M. Abramowitz and I.E. Stegun, Handbook of Mathematical Functions, National Bureau of Standards, Washington, D.C., 1972.

[2] C.L. Dolph, J.B. McLeod, And D. Thoe, The analytic continuation of the resolvent kernel and scattering operator associated with the Schroedinger operator, J. Math. Anal. Appl., 16 (1966), pp. 311-332.

[3] W.F. Donoghue, Distributions and Fourier Transforms, Academic Press, New York, 1969.

[4] G.B. Folland, Real Analysis. Modern Techniques and Their Applications, John Wiley and Sons, New York, 1984. 
[5] R.R. GadyL'SHin, Existence and asymptotics of poles with small imaginary part for the Helmholtz resonator, Russian Math. Surveys, 52 (1997), pp. 1-72.

[6] R.R. Gadyl'shin, On an analog of the Helmholtz resonator in averaging theory, C. R. Acad. Sci. Paris Sér. I Math., 329 (1999), pp. 1121-1126.

[7] S.E. Golowich And M.I. Weinstein, Homogenization expansion for resonances of microstructured photonic waveguides, J. Opt. Soc. Amer. B. Opt. Phys., 20 (2003), pp. 633-647.

[8] S.E. Golowich And M.I. Weinstein, Theory and computation of scattering resonances of microstructures, in Waves in Periodic and Random Media, Contemp. Math. 339, AMS, Providence, RI, 2003, pp. 59-90.

[9] P.D. Hislop AND I.M. SigAL, Introduction to Spectral Theory, Springer-Verlag, New York, 1996.

[10] T. IKEBE, Eigenfunction expansions associated with the Schroedinger operators and their applications to scattering theory, Arch. Rational Mech. Anal., 5 (1960), pp. 1-34.

[11] J. Jasapara, R. Bise, And R. Windeler, Chromatic dispersion measurements in a photonic bandgap fiber, in Proceedings of the Optical Fiber Communication Conference and Exposition, Anaheim, CA, 2002, pp. 519-521.

[12] T. Kato, Perturbation Theory for Linear Operators, 2nd ed., Springer-Verlag, Berlin, New York, 1976.

[13] C. Kittel, Introduction to Solid State Physics, 4th ed., John Wiley and Sons, New York, 1971.

[14] D. Marcuse, Theory of Dielectric Optical Waveguides, 2nd ed., Academic Press, Boston, 1991.

[15] R.B. Melrose, Geometric Scattering Theory, Cambridge University Press, Cambridge, UK, 1995.

[16] P.M. Morse and H. Feshbach, Methods of Theoretical Physics, Vol. I \& II, McGraw-Hill, New York, 1953.

[17] P.M. Morse And K.U. Ingard, Theoretical Acoustics, McGraw-Hill, New York, 1968.

[18] S. Moskow And M. Vogelius, First-order corrections to the homogenized eigenvalues of a periodic composite medium. a convergence proof, Proc. Roy. Soc. Edinburgh Sect. A, 127 (1997), pp. 1263-1299.

[19] L. Poladian, N.A. Issa, And T.M. Monro, Fourier decomposition algorithm for leaky modes of fibres with arbitrary geometry, Opt. Express, 10 (2002), pp. 449-454.

[20] J.K. Ranka, R.S. Windeler, And A.J. Stentz, Visible continuum generation in air-silica microstructure optical fibers with anomolous dispersion at $800 \mathrm{~nm}$, Opt. Lett., 25 (2000), pp. 25-27.

[21] M. Reed And B. Simon, Methods of Modern Mathematical Physics, Vol. I, Academic Press, New York, London, 1972.

[22] M. Reed And B. Simon, Methods of Modern Mathematical Physics, Vol. II, Academic Press, New York, London, 1975.

[23] M. Reed And B. Simon, Methods of Modern Mathematical Physics, Vol. IV, Academic Press, New York, London, 1978.

[24] F. Santosa And M. Vogelius, First-order corrections to the homogenized eigenvalues of a periodic composite medium, SIAM J. Appl. Math., 53 (1993), pp. 1636-1668.

[25] N. Shenk And D. Thoe, Resonant states and poles of the scattering matrix for perturbations of $-\Delta$, J. Math. Anal. Appl., 37 (1972), pp. 467-491.

[26] A.W. Snyder and J. D. Love, Optical Waveguide Theory, Chapman and Hall, London, 1983.

[27] E.M. Stein, Singular Integrals and Differentiability Properties of Functions, Princeton University Press, Princeton, NJ, 1970.

[28] J. Vuckovic, M. Loncar, H. Mabuchi, and A. Scherer, Design of photonic crystal microcavities for cavity QED, Phys. Rev. E, 65 (2001), 016608. 